\title{
Non-Newtonian couple-stress squeeze film behaviour between oscillating anisotropic porous circular discs with sealed boundary
}

\author{
Bilal Boussaha ${ }^{1}$, Mustapha Lahmar ${ }^{1}$, Benyebka Bou-Said ${ }^{2,3}{ }^{*}$, and Hamid Boucherit ${ }^{1}$ \\ ${ }^{1}$ Université 8 Mai 1945 Guelma, Laboratory of Mechanics and Structures (LMS), Mechanical Engineering Department, \\ Guelma, Algeria \\ ${ }^{2}$ Northwestern Polytechnical University, Department of Material Science, Xi'An, PR China \\ ${ }^{3}$ Université de Lyon, CNRS INSA-Lyon, LaMCoS, UMR5259, 69621 Lyon, France
}

Received: 7 October 2019 / Accepted: 13 January 2020

\begin{abstract}
The thrust of this paper is to investigate theoretically the non-Newtonian couple stress squeeze film behaviour between oscillating circular discs based on V. K. Stokes micro-continuum theory. The lubricant squeezed out between parallel porous and rigid facings is supposed to be a concentrated suspension which consists of small particles dispersed in a Newtonian base fluid (solvent). The effective viscosity of the suspension is determined by using the Krieger-Dougherty viscosity model for a given volume fraction of particles in the base fluid. For low frequency and amplitude of sinusoidal squeezing where cavitation as well as turbulence are unlikely, the governing equations including the modified Reynolds equation coupled with the modified Darcy's equation are derived and solved numerically using the finite difference method and a sub-relaxed iterative procedure. The slip velocity at the porous-fluid interface is directly evaluated by means of the modified Darcy's law considering laminar and isothermal squeezing flow. For a given volume fraction, the couple stress effects on the squeeze film characteristics are analyzed through the dimensionless couple stress parameter $\tilde{\ell}$ considering sealed and unsealed boundary of the porous disc. The obtained relevant results reveal that the use of couple stress suspending fluids as lubricants and the effect of sealing the boundary of the porous matrix improves substantially the squeeze film behaviour by increasing the squeeze film force. On the other hand, side leakage flow calculated in the sealed case remains constant in comparison to that of open end (unsealed) porous disc for all values of couple stress parameter and volume fraction of particle.
\end{abstract}

Keywords: Couple stress suspending fluid / squeeze film / anisotropic porous disc / sealed edge / Krieger-Dougherty viscosity model

\section{Introduction}

The squeeze film lubrication mechanism is ubiquitous in many engineering applications such as gears, bearings in rotating and reciprocating machinery, and so on. In such cases, a positive and a negative load can act by turns, and positive and negative squeeze occurs alternatively.

The squeeze effect in lubrication occurs when positive pressure arises in a fluid film between two mutually approaching surfaces. This phenomenon is also called the positive squeeze effect and the lubricating film is called the squeeze film. However, when negative pressure arises in the

\footnotetext{
* e-mail: benyebka.bou-said@insa-lyon.fr
}

fluid film between two mutually receding surfaces, this phenomenon is then called negative squeeze. In this case, fluid is sucked into the gap between the two surfaces contiguous to the film.

In his famous paper on lubrication, Osborne Reynolds [1] stated that the squeeze effect is an important mechanism, together with the wedge and stretching effects, for the self-generation of pressure in a lubricating film, especially when the contribution due to the wedge effect is small compared to the squeeze effect. This is due from the fact that the viscous fluid film cannot be squeezed out instantaneously, so it resists to extrusion which results in build-up of pressure.

Many researchers have investigated theoretically and experimentally the squeeze film phenomenon with a Newtonian fluid among them, we cite Jackson [2], Bujurke 
et al. [3-5], Megat et al. [6], Hvacek [7], Ruggiero et al. [8], Alshehri and Sharma [9], B. J. Hamrock [10], and Yukio Hori [11].

It should be emphasized that there is a huge number of investigations into porous and non-porous squeeze films. For instance, Jurczak [12] proposed bio-bearings models for the squeeze film with one porous layer to study the inertia effect on the pressure field through the use of the MorganCameron [13] approximation for coupling the NavierStokes and Poisson equations. He concluded that the pressure generated in the couple stress fluid is higher than that of the Newtonian fluid. He also found that the pressure decreases when increasing the permeability.

Using the Barus law, Lin et al. [14] analysed the combined effects of viscosity-pressure variations and nonNewtonian couple stresses on the wide parallel rectangular plate squeeze film characteristics. The semi-analytical solution, obtained by means of the small perturbation method, was compared by the authors with that derived in the iso-viscous Newtonian case. They found that the piezo-viscous and couple stresses effects can improve the squeeze film performance characteristics because of the greater carrying capacity obtained for a lower film thickness.

Nabhani et al. [15] presented a new model for combining the effects of couple stresses and porous matrix deformations considering fluid inertia. The authors used the Darcy-Brinkman-Forchheimer generalized model for describing the fluid flow in the porous disc and the simplified thin elastic liner approach to determine the elastic deformation at the porous-fluid interface. They found that the couple stresses and the poro-elasticity of the disc increased the friction coefficient and decreased the load capacity.

Ponnuswamy and Govindaraj [16] analysed squeeze film performances for parallel porous annular disc, with a sinusoidal motion of the upper disc. They derived an analytical expression for pressure by using the FourierBessel series. The obtained results showed that the load capacity increased with increasing the couple stress or the sinusoidal movement amplitude.

Available technical literature reveals that most of the researches done basically consider porous circular or annular discs with unsealed boundary. The MorganCameron approach [13] which ignores any slip effect at the porous-fluid interface has been often adopted to take into account the porosity effects.

Moreover, the slip phenomenon at the porous-fluid interface has been incorporated by means of some more complex models such as Beavers et al. [17] and Brinkman [18]. An elaborate discussion on the choice of slip parameters and the validity of the different slip-flow boundary conditions is given in [19].

The aim of this paper is to investigate more particularly the non-Newtonian couple-stress squeeze film behaviour between two oscillating circular discs with either sealed or unsealed boundary with a view to application for polluted mechanisms. The present investigation is based on the V. K. Stokes micro-continuum theory which accounts for couple-stresses due to the presence of small particles in the suspending fluid.
The upper disc is impermeable and mobile, while the lower one is assumed to be a stationary anisotropic porous medium. The effective viscosity of the suspension is determined by using the Krieger-Dougherty viscosity model for a given volume fraction of particles in the base fluid.

It should be noted that under dynamic loading conditions (i.e., for intense sinusoidal motion), cavitation may occur as the upper and lower surfaces move away from each other during part of the harmonic squeeze motion (i.e., for negative squeeze phase) executed by the upper disc. As the surfaces contiguous to the film separate, this creates sub-cavity pressures within the film gap which are less than the absolute atmospheric pressure. If the sub-cavity pressures fall below a threshold cavitation pressure, the fluid film will break, i.e., cavitate. The cavitation may be gaseous, vaporous, or some combination thereof.

Considering low amplitude and frequency sinusoidal squeezing where cavitation as well as turbulence is unlikely, the governing equations including the modified Reynolds equation coupled with the modified Darcy's equation are derived for a non-Newtonian suspending couple stress fluid model with variable viscosity considering slip effects at the porous-fluid interface. The slip velocity is directly computed by means of the modified Darcy's law for low squeeze Reynolds numbers, i.e. for laminar squeezing flows. The squeeze film performance characteristics are then obtained from the numerical solution of the two governing equations using the finite difference method and a sub-relaxed iterative procedure. For a given volume fraction, the couple stress effects on the squeeze film characteristics will be analyzed for a range of the dimensionless couple stress parameter $\tilde{\ell}$ considering sealed and unsealed boundary of the porous disc.

\section{Theoretical formulation}

The study of lubrication of such mechanism is schematically shown in Figure 1 by two horizontal discs, separated by a polluted film. The effect of squeezing between two discs is analyzed when one rigid disc which approaches with uniform velocity to the other disc has a porous facing, without neglecting the sliding motion on them. The lubricant fluid in the film region is supposed to be a couple stress fluid $[20,21]$. When the body forces, and the body moments are absent, the momentum equation and the continuity equation for inertialess incompressible couple stress fluid take the forms [22,23]:

$$
\begin{gathered}
\vec{\nabla} p=-\mu_{s} \overrightarrow{\boldsymbol{r o t}}(\overrightarrow{\boldsymbol{r o t}} \vec{V})-\eta \overrightarrow{\boldsymbol{r o t}}(\overrightarrow{\boldsymbol{r o t}}(\overrightarrow{\boldsymbol{r o t}}(\overrightarrow{\boldsymbol{r o t}} \vec{V}))) \\
\vec{\nabla} \cdot \vec{V}=0
\end{gathered}
$$

where $\overrightarrow{\boldsymbol{V}}=u \overrightarrow{\boldsymbol{e}}_{\boldsymbol{r}}+v \overrightarrow{\boldsymbol{e}}_{\theta}+w \overrightarrow{\boldsymbol{e}}_{z}$ is the flow velocity vector $p$, the pressure, $\mu_{s}$ is the effective viscosity of suspension fluid, and $\eta$ is the material constant responsible for the couple stress property. The dimensional equation of the new 


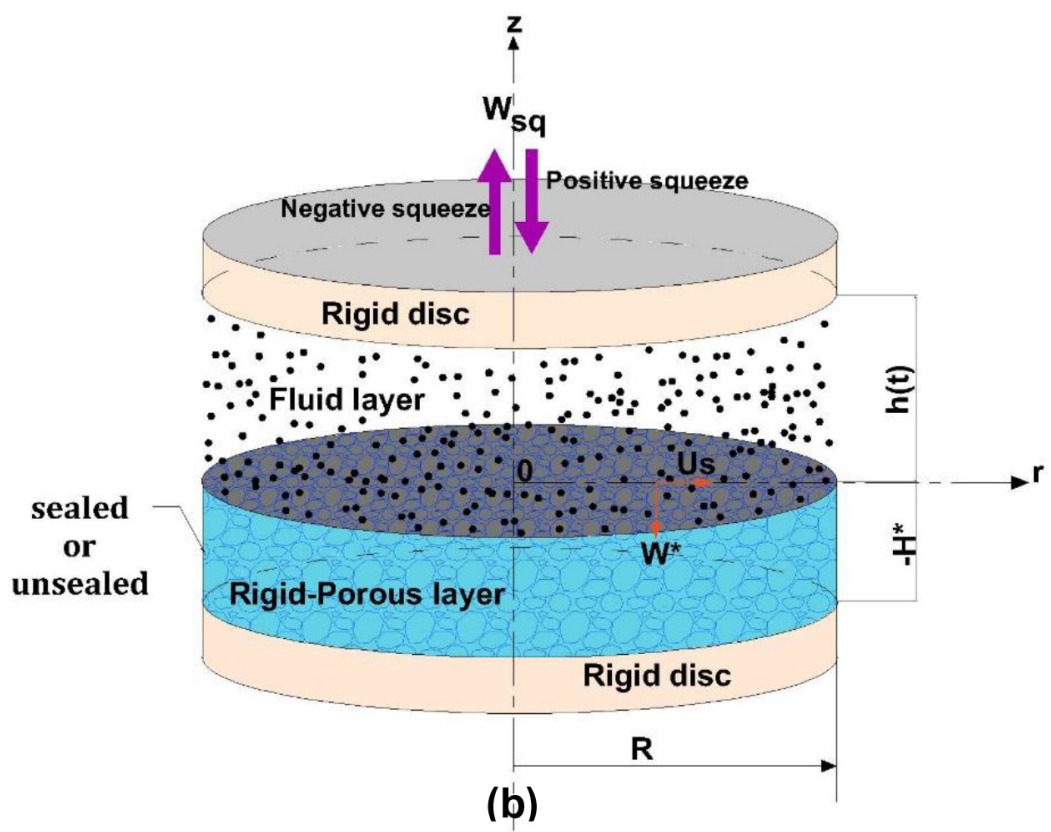

Fig. 1. Geometry model.

material constant is that of momentum, i.e. $\eta=\mathrm{MLT}^{-1}$. Some experiments to determine this material constant were given in reference [21].

To calculate the effective viscosity, Krieger-Dougherty [24] in 1959 derived an equation for the dynamic viscosity covering the full range of volume fraction in the base fluid (solvent):

$$
\mu_{s}=\mu_{b f}\left(1-\frac{\phi}{\phi_{m}}\right)^{-[\bar{\eta}] \phi_{m}}
$$

where $\mu_{\mathrm{bf}}$ is the viscosity of base fluid, $\phi$ is the volume fraction of particle in base fluid, $\phi_{m}$ is the maximum particle packing fraction, which varies from 0.495 to 0.54 under quiescent conditions, and is approximately 0.605 at high shear rates $[25,26]$ and $[\bar{\eta}]$ is the intrinsic viscosity, whose typical value for mono disperse suspensions of hard spheres is 2.5 [27].

Typical variations of the relative viscosity $\frac{\mu_{s}}{\mu_{s}}$ with the volume fraction $\phi$ are presented in Figure 2 using the Krieger-Dougherty relationship (3) and the well-known classical Einstein model [28].

Using the classical assumption of thin film lubrication $[29,30]$ and when the problem is considered axisymmetric (i.e., $\frac{\partial}{\partial \theta}=0$ equations (1) and (2) in cylindrical coordinates $(r, \theta$, and $z)$ reduce to

$$
\begin{gathered}
\frac{\partial p}{\partial r}=\mu_{s} \frac{\partial^{2} u}{\partial z^{2}}-\eta \frac{\partial^{4} u}{\partial z^{4}} \\
\frac{\partial p}{\partial \theta}=\frac{\partial p}{\partial z}=0 \\
\frac{1}{r} \frac{\partial(r u)}{\partial r}+\frac{\partial w}{\partial z}=0
\end{gathered}
$$

where $u, w$ are the components of the flow velocity vector in the radial and axial directions, respectively. The velocity components in the porous matrix are given for an isothermal laminar flow by the modified Darcy's law, which accounts for the couple stress effects [31]:

$$
\begin{aligned}
& u^{*}=-\alpha_{r}\left(\frac{\partial p^{*}}{\partial r}\right), \\
& w^{*}=-\alpha_{z}\left(\frac{\partial p^{*}}{\partial z}\right),
\end{aligned}
$$

where $u^{*}$ and $w^{*}$ are the radial and axial components of the flow velocity in the porous layer, respectively, $p^{*}$ is the pressure field in the porous layer, $\alpha_{r}=\frac{\kappa_{r}}{\mu_{s}\left(1-\beta_{r}\right)}$ and $\alpha_{z}=\frac{\kappa_{z}}{\mu_{s}\left(1-\beta_{z}\right)}$ are the permeability parameters of the anisotropic porous matrix in the $r$ and $z$ directions, respectively. $\beta_{r}$ and $\beta_{z}[32,33]$ appearing in the relationships of $\alpha_{r}$ and $\alpha_{z}$ which represent the microstructure size to the pore size ratios are defined by the following relationships :

$$
\begin{gathered}
\beta_{r}=\frac{\eta / \mu_{s}}{\kappa_{r}} \\
\beta_{z}=\frac{\eta / \mu_{s}}{\kappa_{z}}
\end{gathered}
$$

where $\eta / \mu_{s}$ has a dimension of the length squared, and $\kappa_{r}$ and $\kappa_{z}$ are the permeability coefficients in the $r$ and $z$ directions, respectively. 


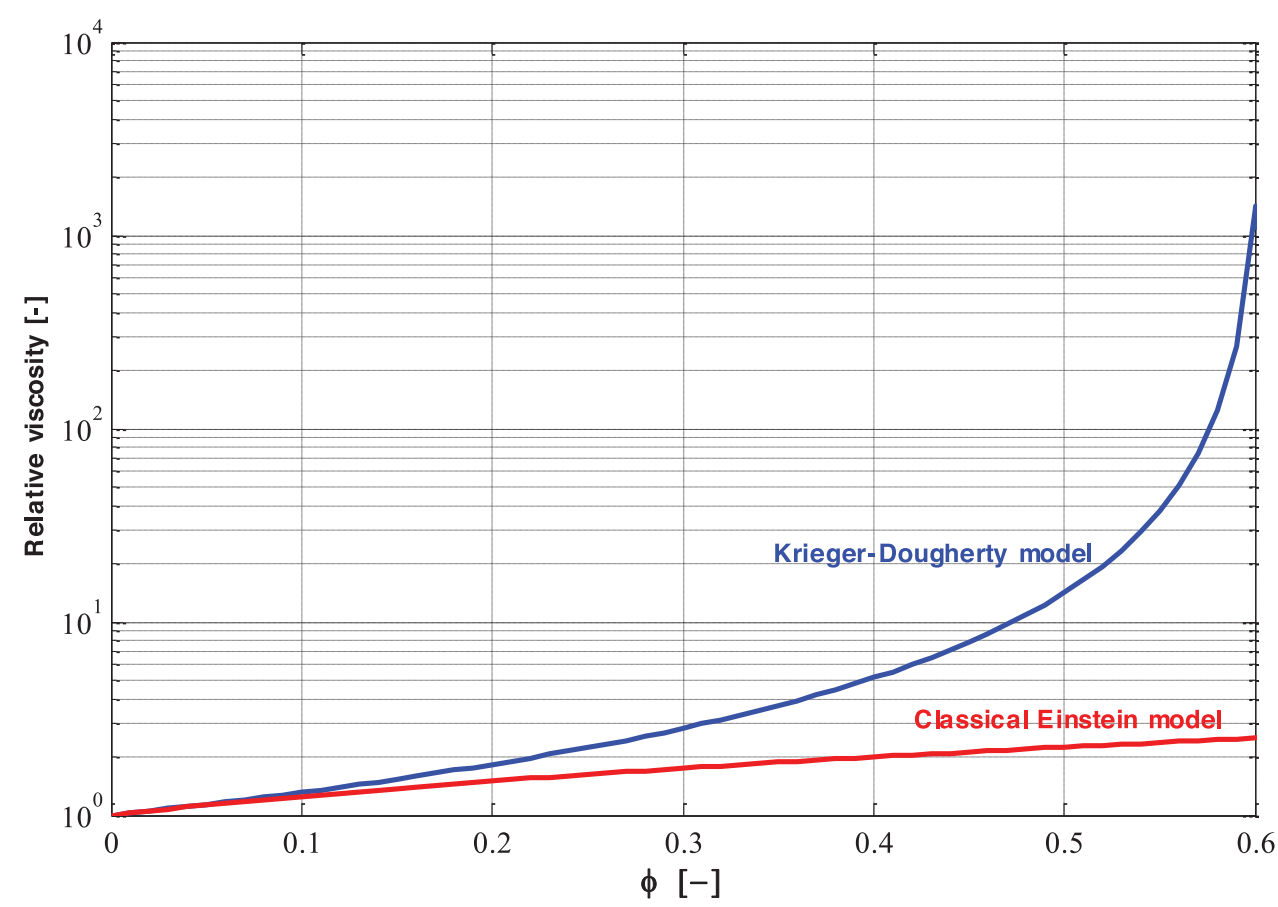

Fig. 2. Comparison of the Krieger-Dougherty viscosity model with the conventional viscosity model of Einstein $\left(\frac{\mu_{s}}{\mu_{\mathrm{bf}}}=1+[\bar{\eta}] \phi\right)$ over a range of volume fractions.

The pressure field in the porous layer must satisfy the following modified Laplace equation written for a couple stress fluid:

$$
\alpha_{r} \frac{1}{r} \frac{\partial}{\partial r}\left(r \frac{\partial p^{*}}{\partial r}\right)+\alpha_{z} \frac{\partial^{2} p^{*}}{\partial z^{2}}=0
$$

If we assume that the film thickness as a function of time, $t$, in the case of positive and negative squeeze changes in a sinusoidal manner, in this case, the film thickness $h(t)$ and the squeeze velocity $W_{\mathrm{sq}}=\dot{h}(t)$ will be given by:

$$
\begin{gathered}
h(t)=h_{0}+h_{a}(\cos (2 \pi f t)-1), \\
W_{\mathrm{sq}}=-2 \pi f h_{a} \sin (2 \pi f t),
\end{gathered}
$$

where $h_{0}$ is an initial film thickness, $h_{a}$ is the amplitude, and $f$ is the sinusoidal motion frequency.

Typical variations of the dimensionless film thickness $\tilde{h}$ and the dimensionless squeeze velocity $\tilde{W}_{\text {sq }}$ with dimensionless time $\tilde{t}=t f$ obtained from equations (11) for $\tilde{h}_{a}=0.2$ are shown in Figure 3 .

The relevant boundary conditions for the velocity components are:

- At the upper surface $z=h(t)$

$$
u=0, w=\frac{d h(t)}{\mathrm{d} t}=W_{\mathrm{sq}}, \frac{\partial^{2} u}{\partial z^{2}}=0
$$

- At the fluid-porous interface $z=0$

$$
u=U_{s}=\left.u^{*}\right|_{z=0}, w=\left.w^{*}\right|_{z=0,} \frac{\partial^{2} u}{\partial z^{2}}=0 .
$$

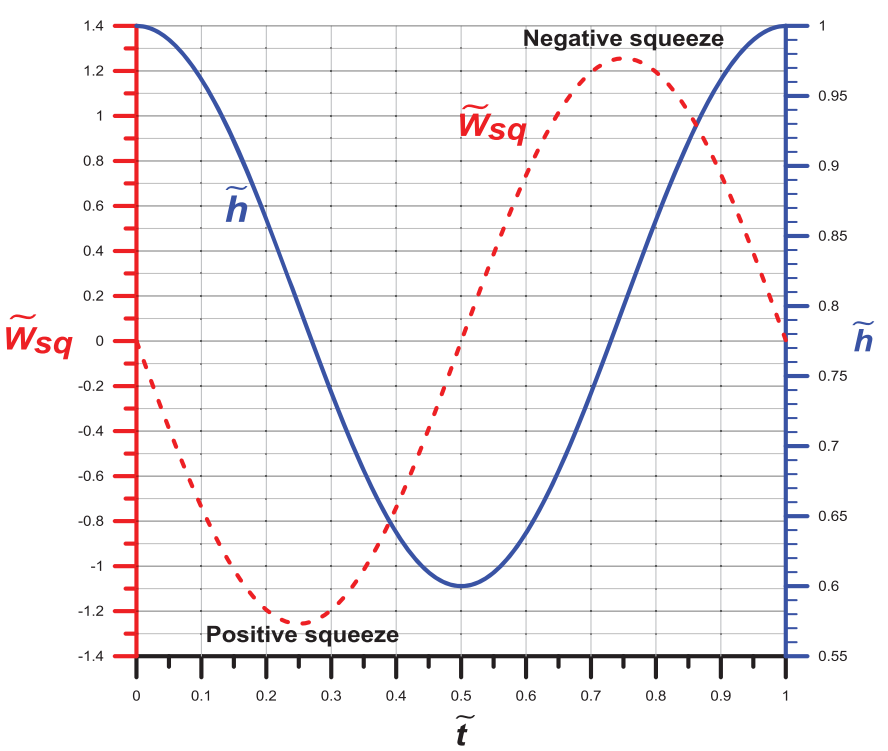

Fig. 3. Time variations of normalized film thickness and squeeze velocity.

The relevant boundary conditions for the pressure are:

$$
\begin{aligned}
& p=0 \text { (ambient pressure) and } \\
& \times\left\{\begin{array}{cc}
\frac{\partial p^{*}}{\partial r}=0 & \text { (sealed case) } \\
& \text { or }
\end{array} \quad \text { at } r=R,\right.
\end{aligned}
$$




$$
\begin{gathered}
\frac{\partial p}{\partial r}=0 \text { and } \frac{\partial p^{*}}{\partial r}=0 \text { at } r=0(\text { axisymmetric field }), \\
p=p^{*} \text { at } z=0(\text { continuity of pressure }) \\
\frac{\partial p^{*}}{\partial z}=0 \text { at } z=-H^{*}(\text { rigid substrate })
\end{gathered}
$$

where $H^{*}$ is the thickness of the porous layer.

The solution of equation (4) subjected to the boundary conditions (12) is:

$$
\begin{aligned}
u(r, z)= & \frac{1}{2 \mu_{s}} \frac{\partial p}{\partial r}\left[z(z-h)+2 l^{2}\left(1-\frac{\cosh \left(\frac{2 z-h}{2 l}\right)}{\cosh \left(\frac{h}{2 l}\right)}\right)\right] \\
& +U_{s}\left(1-\frac{z}{h}\right)
\end{aligned}
$$

where $l=\sqrt{\eta / \mu_{s}}$ is the couple stress parameter, $U_{s}$ is the slip velocity at the fluid-porous layer interface $(z=0)$ given for a laminar squeezing flow by:

$$
U_{s}=-\left.\alpha_{r}\left(\frac{\partial p^{*}}{\partial r}\right)\right|_{z=0}
$$

This is a direct incorporation of the slip effect at the porous-fluid interface.

The fluid velocity $w$ in the $z$-direction can be obtained numerically using the finite difference method from the derivation of the continuity equation (6) with respect to the $z$-variable, i. e.

$$
\frac{\partial^{2} w}{\partial z^{2}}=-\frac{1}{r} \frac{\partial}{\partial r}\left(r \frac{\partial u}{\partial z}\right)
$$

Integrating the continuity equation (6) through the fluid film thickness and using the modified Darcy's law (14) and the boundary conditions (12) for the axial fluid velocity $w$ we get the modified Reynolds' equation:

$$
\frac{G(h, l)}{12 \mu_{s}} \frac{1}{r} \frac{\partial}{\partial r}\left(r \frac{\partial p}{\partial r}\right)=\frac{d h}{\mathrm{~d} t}-\left.w^{*}\right|_{z=0}+\frac{h}{2}\left(\frac{\partial U_{s}}{\partial r}+\frac{U_{s}}{r}\right),
$$

where

$$
G(h, l)=h^{3}-12 l^{2} h+24 l^{3} \tanh \left(\frac{h}{2 l}\right) .
$$

Substituting equation (8) into equation (17), a final form of the modified Reynolds equation can then be derived:

$$
\begin{aligned}
\frac{G(h, l)}{12 \mu_{s}} \frac{1}{r} \frac{\partial}{\partial r}\left(r \frac{\partial p}{\partial r}\right)= & \frac{d h}{\mathrm{~d} t}+\left.\alpha_{z}\left(\frac{\partial p^{*}}{\partial z}\right)\right|_{z=0} \\
& +\frac{h}{2}\left(\frac{\partial U_{s}}{\partial r}+\frac{U_{s}}{r}\right) .
\end{aligned}
$$

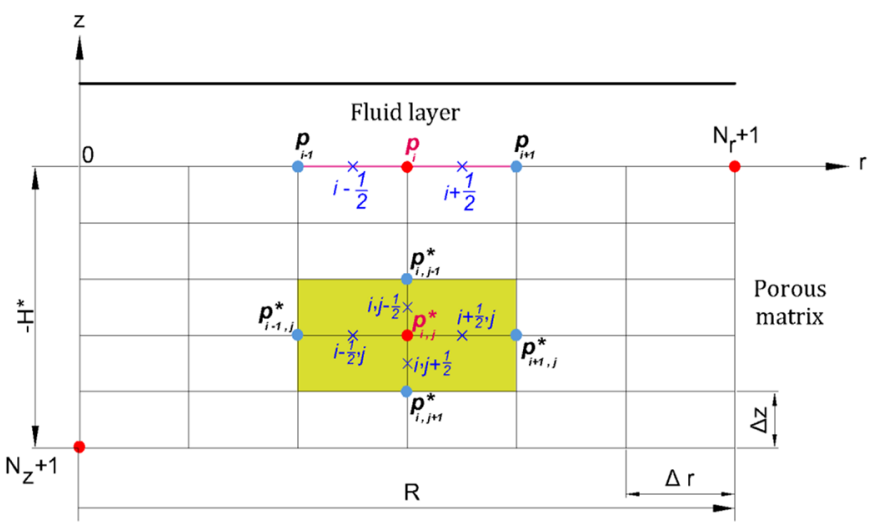

Fig. 4. Axisymmetric finite difference grid of the fluid-film and porous media.

$$
\text { Recall that } U_{s}=-\left.\alpha_{r}\left(\frac{\partial p^{*}}{\partial r}\right)\right|_{z=0}
$$

\section{Numerical formulation}

The modified Reynolds equation (19) and the modified Laplace equation (10) are coupled at the porous interface by the continuity of pressure and velocities, and solved numerically by the finite difference method (Fig. 4).

The finite difference equations resulting from the discretization process of the two governing equations are given below

- For the modified Reynolds' equation

$$
p_{i}=A_{1} p_{i+1}+A_{2} p_{i-1}+A_{3}
$$

where:

$$
\begin{aligned}
& A_{0}=\frac{G(h, l)}{12 \mu_{s}}\left(r_{i+\frac{1}{2}}+r_{i-\frac{1}{2}}\right) / \Delta r^{2}, \\
& A_{1}=\left(r_{i+\frac{1}{2}} \frac{G(h, l)}{12 \mu_{s}}\right) /\left(A_{0} \Delta r^{2}\right), \\
& A_{2}=\left(r_{i-\frac{1}{2}} \frac{G(h, l)}{12 \mu_{s}}\right) /\left(A_{0} \Delta r^{2}\right),
\end{aligned}
$$

$$
A_{3}=\left(-r_{i} \frac{d h}{\mathrm{~d} t}-\left.r_{i} \alpha_{z}\left(\frac{\partial p^{*}}{\partial z}\right)\right|_{i, 0}-\frac{h}{2}\left(\left.r_{i} \frac{\partial U_{s}}{\partial r}\right|_{i}+U_{s_{i}}\right)\right) / A_{0} .
$$

- For the modified Darcy's equation

$$
p_{i, j}^{*}=C_{1} p_{i+1, j}^{*}+C_{2} p_{i-1, j}^{*}+C_{3}\left(p_{i, j+1}^{*}+p_{i, j-1}^{*}\right)
$$

where:

$$
C_{0}=\left[\alpha_{r}\left(r_{i+\frac{1}{2}}+r_{i-\frac{1}{2}}\right) / \Delta r^{2}\right]+\left[2 r_{i} \alpha_{z} / \Delta z^{2}\right],
$$




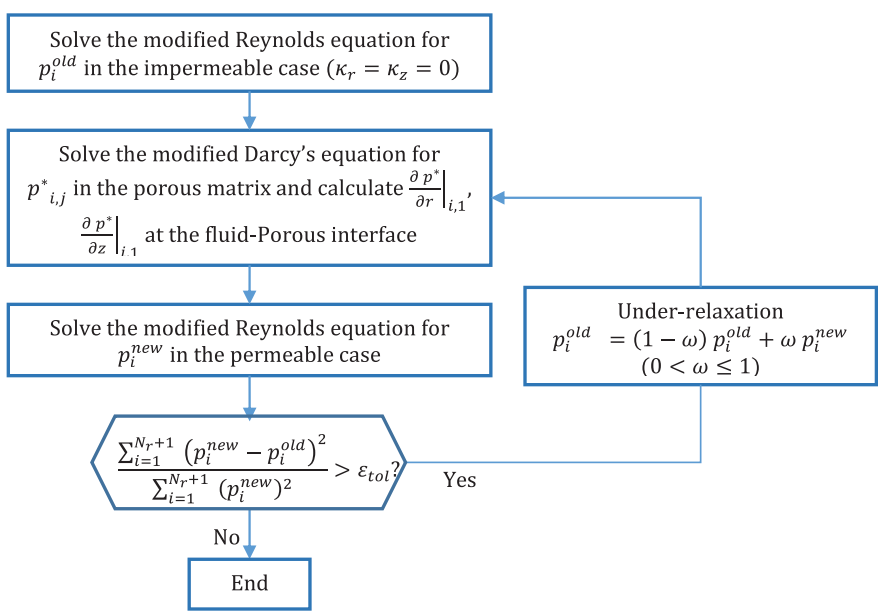

Fig. 5. Iterative scheme for solving the coupled Reynolds-Darcy equations.

$$
\begin{gathered}
C_{1}=r_{i+\frac{1}{2}} \alpha_{r} /\left(C_{0} \Delta r^{2}\right), \\
C_{2}=r_{i-\frac{1}{2}} \alpha_{r} /\left(C_{0} \Delta r^{2}\right), \\
C_{3}=r_{i} \alpha_{z} /\left(C_{0} \Delta z^{2}\right) .
\end{gathered}
$$

The obtained system of linear algebraic equations for both governing differential equations is solved numerically by means of the iterative Gauss-Seidel method with overrelaxation factor $\omega_{G S}$ whose value ranges from 1 to 2 (i.e., $1 \leq \omega_{\mathrm{GS}}<2$ ) [34].

Figure 5 illustrates the general procedure for the iterative process used at each time step. To obtain a converged solution for the system of coupled partial differential equations an iteration scheme is required. For the first iteration, the modified Reynolds equation (20) is solved for $p_{i}^{\text {old }}$ without the influence of the porous matrix. The obtained fluid-film pressure field can then be used as a boundary condition at the fluid-porous interface to solve the modified Darcy's equation (22) for $p_{i, j}^{*}$ in the porous medium. The calculated pressure $p_{i, j}^{*}$ serves to compute the radial and axial pressure gradients $\left.\frac{\partial p^{*}}{\partial r}\right|_{i, 1}$ and $\left.\frac{\partial p^{*}}{\partial z}\right|_{i, 1}$ and therefore velocity components $u_{i, 1}^{*}$ and $w_{i, 1}^{*}$ at the interface. It is now possible to solve a second time the modified Reynolds equation (20) for $p_{i}^{\text {new }}$ taking into account the terms related to porosity appearing on the RHS of the fluidfilm equation. With the resulting pressure the process of calculation returns to solving the modified Darcy's equation (22). This procedure is repeated until a converged solution has been found which results in obtaining two successive fluid pressure fields very close.

To avoid numerical oscillations it is important to limit the influence per iteration of the porous matrix into the pressure solution of the Reynolds equation (20). To this end an under-relaxation parameter is introduced, which has to be chosen such that convergence is guaranteed, but the calculation time still remains acceptable.
Once the convergence of process is obtained we can calculate the hydrodynamic performance characteristics such as the lift load, the drag force, the leakage flow, and the power loss.

\section{Hydrodynamic performance characteristics}

The instantaneous leakage flow of lubricant is calculated by the following relation:

$$
Q=2 \pi R h\langle u\rangle
$$

where $\langle u\rangle=\frac{1}{h} \int_{0}^{h} u(r=R, z) d z$

Substituting the expression for $u$ from equation (14) into the leakage flow equation (24) and then integrating over the film thickness yields:

$$
Q=-2 \pi R\left(\left.\frac{G(h, l)}{12 \mu_{s}} \frac{\partial p}{\partial r}\right|_{r=R}-\frac{h U_{s}}{2}\right) .
$$

By integrating the film pressure over the fluid layer, we express the instantaneous load carrying capacity so-called lift force $W$ of the squeeze film as follows:

$$
W=2 \pi \int_{0}^{R} p(r) r d r
$$

for couple stress fluid, the parietal shear stress $\tau_{r z}$ given by:

$$
\tau_{r z}=\left.\mu_{s} \frac{\partial u}{\partial z}\right|_{z=0}-\left.\eta \frac{\partial^{3} u}{\partial z^{3}}\right|_{z=0} .
$$

By using the expression of $u$ and boundary conditions, we find:

$$
\tau_{r z}=-\left(\frac{h}{2} \frac{\partial p}{\partial r}+\mu_{s} \frac{U_{s}}{h}\right) .
$$

Thus, the friction force $F_{f}$ can be calculated by integrating the shear stress :

$$
F_{f}=-2 \pi \int_{0}^{R}\left(\frac{h}{2} \frac{\partial p}{\partial r}+\mu_{s} \frac{U_{s}}{h}\right) r d r .
$$

The total power loss can be calculated by:

$$
\mathcal{P}=2 \pi[\underbrace{\int_{0}^{h} \int_{0}^{R} \Phi_{1} r d r d z}_{\text {Newtonian contribution }}+\underbrace{\int_{0}^{h} \int_{0}^{R} \Phi_{2} r d r d z}_{\text {Non-Newtonian contribution }}]
$$

where $\Phi_{1}$ and $\Phi_{2}$ are the dissipation functions expressed by:

$$
\Phi_{1}=\mu_{s}\left[\left(\frac{\partial u}{\partial z}\right)^{2}+\left(\frac{\partial w}{\partial z}\right)^{2}\right] \approx \mu_{s}\left(\frac{\partial u}{\partial z}\right)^{2}
$$




$$
\Phi_{2}=\eta\left[\left(\frac{\partial^{2} u}{\partial z^{2}}\right)^{2}+\left(\frac{\partial^{2} w}{\partial z^{2}}\right)^{2}\right] \approx \eta\left(\frac{\partial^{2} u}{\partial z^{2}}\right)^{2}
$$

After integration with respect to $\mathrm{z}$, we get the final form of total power loss:

$$
\mathcal{P}=2 \pi[\underbrace{\int_{0}^{R} \frac{G(h, l)}{12 \mu_{s}}\left(\frac{\partial p}{\partial r}\right)^{2} r d r}_{\text {Hagen-Poiseuille power }}+\underbrace{\int_{0}^{R} \frac{\mu_{s} U_{s}^{2}}{h} r d r}_{\text {slip induced power }}] .
$$

\section{Results and discussion}

\subsection{Validation}

The calculation flowchart employed in the present analysis (Fig. 5) is validated by comparing the numerical results obtained by the developed computer code with the simplified analytical solution based on the MorganCameron approximation (see Appendix A for details). The obtained results are displayed and compared in Figure 6 . The calculations are performed using the data reported in Table 1 for $\alpha_{r}=0$. Good agreement is observed between numerical and analytical results and we may conclude that the proposed method of solution used in the present analysis is validated for a small porous layer thickness.

\subsection{Parametric study}

The numerical model developed is used to investigate the effects of couple stresses for given values of volume fraction on hydrodynamic performance characteristics over one cycle of oscillating squeeze motion of sealed or unsealed porous circular discs. The couple stress effects are controlled by the dimensionless parameter $\tilde{\ell}$ varying from 0 . (Newtonian case) to 0.4. The data used in this investigation are given in Table 1.

Figure 7 shows the axisymmetric positive squeeze film pressure $p$ as a function of non-dimensional radial coordinate $\tilde{r}$ at dimensionless time $\tilde{t}=0.3$ for various values of the couple stress parameter $\tilde{\ell}$. It is observed that the effect of couple stresses increases the squeeze film pressure as compared to the Newtonian case $(\tilde{\ell}=0)$ and this increase is more pronounced for larger values of $\tilde{\ell}$. This effect has also been confirmed by the research authors $\mathrm{V}$. Ponnuswamy and S. Govindaraj [16].

In addition, for a same value of the couple stress parameter the pressure calculated for sealed configuration is greater than that obtained for unsealed case especially for higher values of $\tilde{\ell}$. Indeed, the highest rate of increase of central-pressure is about $3 \%$ in Newtonian case and $10 \%$ in non-Newtonian case when $\tilde{\ell}=0.4$. Furthermore, the distributions of pressure within the fluid film and porous disc for sealed and unsealed configurations obtained at $\tilde{t}=0.3$ and $\tilde{\ell}=0.2$ have the same patterns except in the region near of the porous disc outer edge as clearly illustrated in Figure 8.

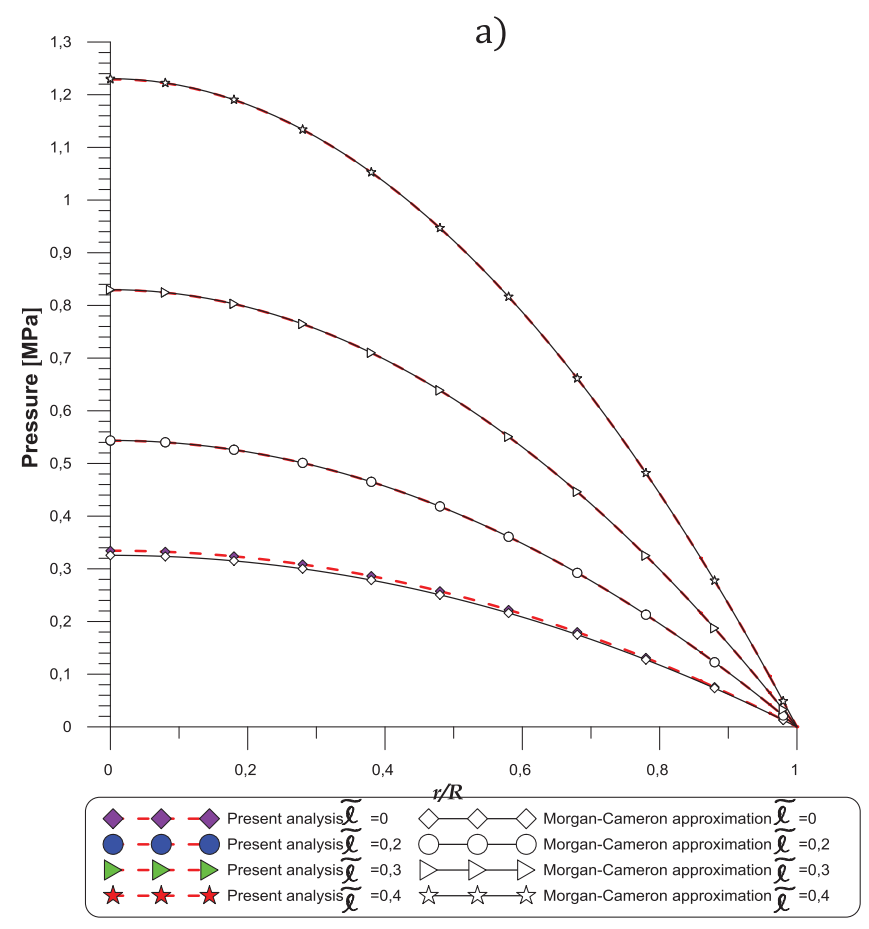

b)
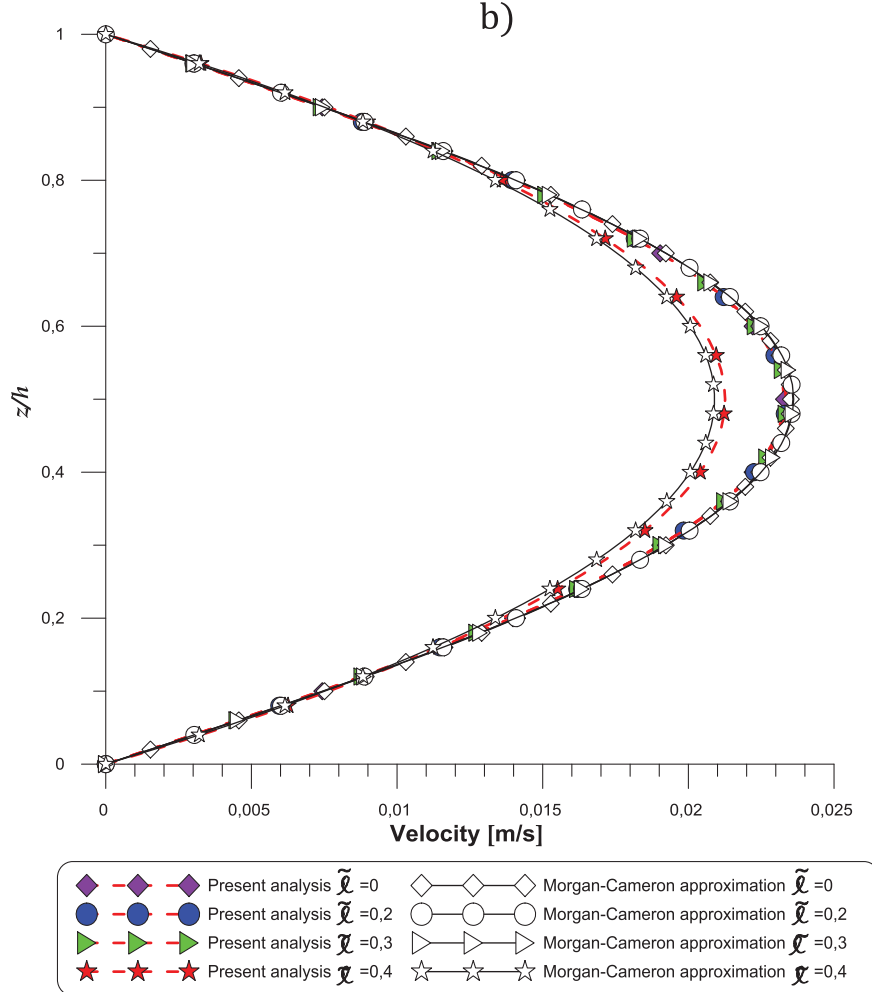

Fig. 6. Comparison of pressure and radial velocity profiles calculated by the iterative numerical procedure and the MorganCameron approximation. (a) Pressure profiles, (b) radial velocity profiles.

The influence of the couple stress parameter $\tilde{\ell}$ in the axisymmetric radial fluid velocity profile $u$ at the edge $(r=R)$ or $\tilde{t}=0.3$ is depicted in Figure 9. The increase of the couple stress parameter $\tilde{\ell}$ results in the reduction of the 
Table 1. Geometrical and physical data.

\begin{tabular}{|c|c|c|c|c|}
\hline Parameters & Symbols & Numerical values & Units & References \\
\hline Initial film thickness & $h_{0}$ & $10^{-5}$ & {$[\mathrm{~m}]$} & {$[43]$} \\
\hline Porous disc thickness & $H^{*}$ & $22 \times 10^{-4}$ & {$[\mathrm{~m}]$} & {$[44]$} \\
\hline Permeability parameter in the $\boldsymbol{r}$-direction & $\alpha_{r}$ & $2.2 \times 10^{-13}$ & {$\left[\mathrm{~m}^{4} / N . \mathrm{s}\right]$} & {$[45]$} \\
\hline Volume fraction of particle & $\phi$ & $0,0.1,0.3$ & {$[-]$} & {$[46]$} \\
\hline $\begin{array}{l}\text { Material constant responsible } \\
\text { for the couple stress property }\end{array}$ & $\eta$ & $\begin{array}{l}0,4 \times 10^{-14}, 9 \times 10^{-14} \\
1.6 \times 10^{-13}\end{array}$ & {$[N . \mathrm{s}]$} & {$[20]$} \\
\hline Dimensionless couple stress parameter & $\tilde{\ell}$ & $0,0.2,0.3,0.4$ & {$[-]$} & - \\
\hline
\end{tabular}

radial velocity in the fluid layer compared to the Newtonian case. This reduction is due to the non-Newtonian couple stress behaviour which provides more resistance to fluid flow. The obtained results are qualitatively in good accordance with those obtained by Nabhani et al. [15] who used a CFD approach, and by Nabhani et al. [35] who used Darcy-Brinkman equations for describing the fluid flow within the porous layer. It is also observed that there are no significant differences of couple stress parameter effect on the radial velocity profile for the sealed configuration.

Figure 10 represents axisymmetric velocity fields and streamlines at $\tilde{t}=0.3$ and for $\tilde{\ell}=0.2$ obtained in the fluid film and porous disc. We can see that the fluid is accelerated in the low pressure zone (Fig. 8) and the velocity profiles in the film gap have a parabolic shape whereas the axisymmetric velocity profiles are nearly uniform across the porous disc for both sealed and unsealed cases. In the same figure, we can find the same flow patterns (streamlines) in the porous disc for both sealed and unsealed configurations except in the outlet region.

The effects of the couple stress parameter $\tilde{\ell}$ in the shear stress distributions across the film thickness, $\tau_{r z}$, are shown in Figure 11 at $\tilde{t}=0.3$ and for $r=R$. It is observed that the greater the couple stress parameter $\tilde{\ell}$ the greater is the shear stress which results in an increased resistance to the flow. As expected, the shear stresses calculated for both
Newtonian and non-Newtonian cases are maximum at the walls and vanish at mid film gap. On the other hand, the parietal shear stresses for sealed configuration are greater than those obtained for the unsealed one. Therefore, the sealed case leads to the greater drag force.

Figure 12 presents the axisymmetric distribution of the squeeze film pressure $p$ during one cycle of oscillating squeeze motion obtained for $\tilde{\ell}=0.2$ (couple stress fluid case) and different values of volume fraction $\phi$. The positive and negative pressures are obtained during positive and negative squeeze motions, respectively. It can be seen that the pressure $p$ generated in the fluid layer is parabolic and is symmetric about the horizontal axis because the fluid inertia forces are ignored as stated previously. Similar trends have been found experimentally by Y. Hori [11] (Chap. 7) for the Newtonian case as well as by J. R. Lin [36] for oscillating circular squeeze films considering nonNewtonian couple stresses through a theoretical investigation.

The pressure vanishes at $\tilde{t}=0.5$ corresponding to $t=0.5 \mathrm{~s}$ for which the squeeze velocity becomes zero as depicted in Figure 3. Furthermore, the squeeze film pressure at $\tilde{t}=0.3$ i.e., during the positive squeezing motion, is greater than the squeeze film pressure predicted at $\tilde{t}=0.2$ since the squeeze velocity $W_{\mathrm{sq}}$ is higher and the film thickness is lower. Furthermore, it is found that the 


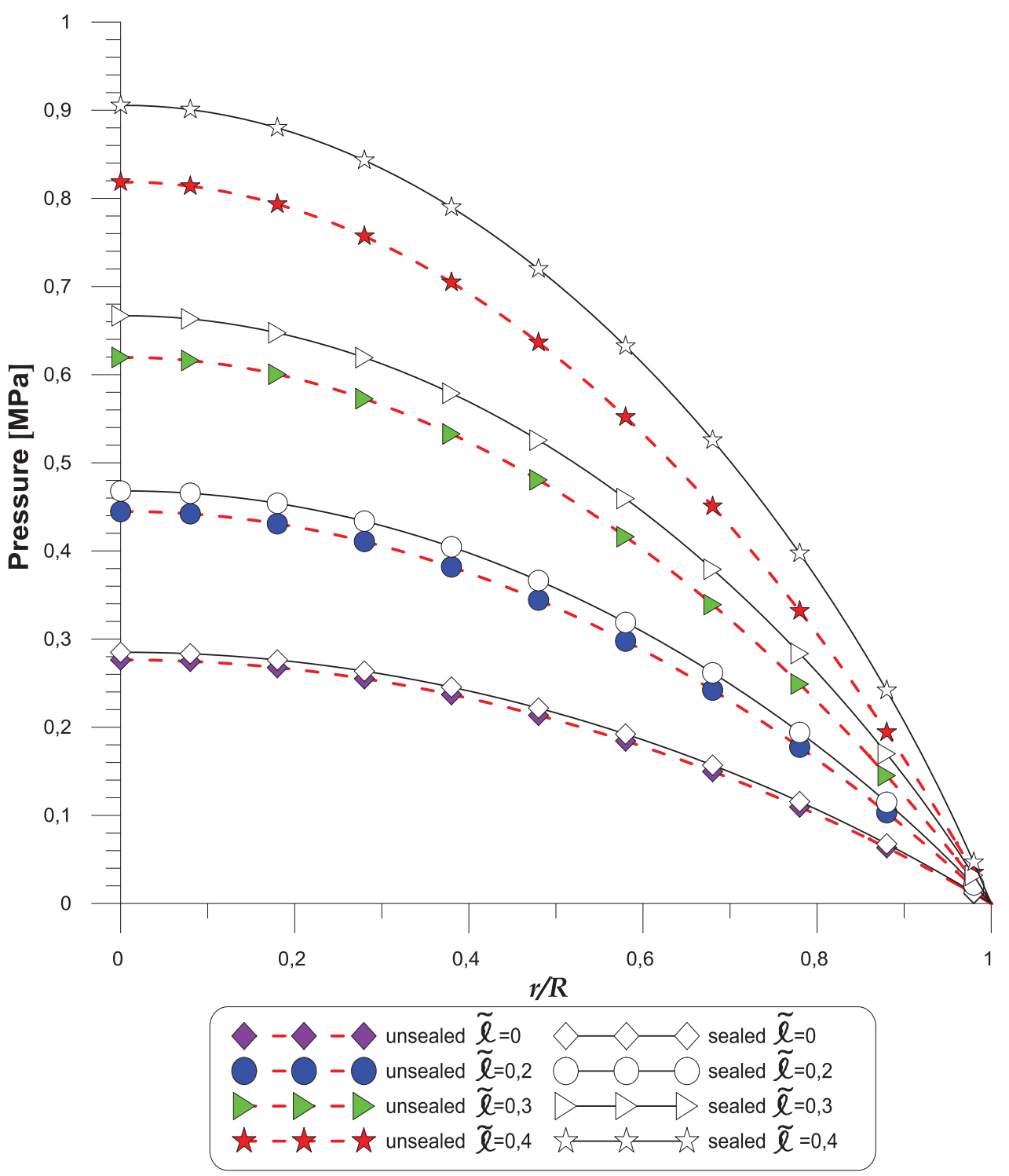

Fig. 7. Distributions of the film pressure $\boldsymbol{p}$ calculated for different values of the couple stress parameter $\tilde{\ell}$ for both sealed and unsealed configurations at $\tilde{\boldsymbol{t}}=0.3$ and for $\phi=0$.

squeeze film pressure increases significantly when increasing the volume fraction of particles in the base fluid. The rate of increase at $\tilde{t}=0.3$ is about $5 \%$ or $\phi=0$ and $9 \%$ or $\phi=0.3$. Accordingly, the suspending fluid gives an enhancement of the carrying load than the non-suspending fluid, i.e. without particles. On the other hand, the squeeze film pressure calculated for the sealed case is much larger than that obtained in the unsealed case for both positive and negative squeeze.

The concentration effect on the axisymmetric radial velocity profile at the edge $(r=R)$ during one cycle of oscillating squeeze motion calculated for $\tilde{\ell}=0.2$ are displayed in Figure 13. It seems that there is no significant influence of the concentration on the velocity distributions for both sealed and unsealed cases. For all values of the concentration, we obviously observe that the radial velocities calculated in the sealed case are more important than those obtained in the unsealed case for both positive and negative squeeze.

Figure 14 displays the axisymmetric shear stress profile $\tau_{r z}$ during one cycle of oscillating squeeze motion computed for $\tilde{\ell}=0.2$ at the outlet of contact $r=R$ for different values of volume fraction $\phi$. It is observed that the shear stress increases significantly with increasing of the volume fraction. As depicted in the figure, the maximum shear stress occurs at the walls. This increase can be explained by the viscosity increase of the suspension. As noted earlier for a fixed time (Fig. 11) the shear stress $\tau_{r z}$ calculated for sealed configuration is greater than that obtained for the unsealed configuration and this for both positive and negative squeezing motions. 


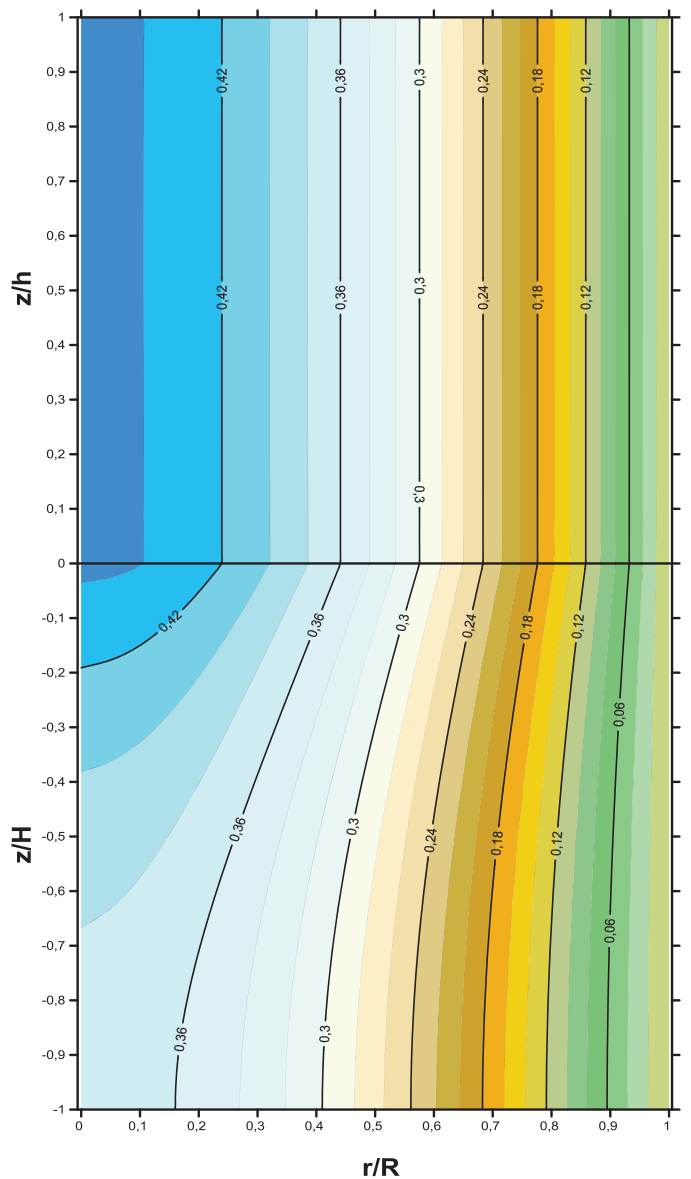

unsealed

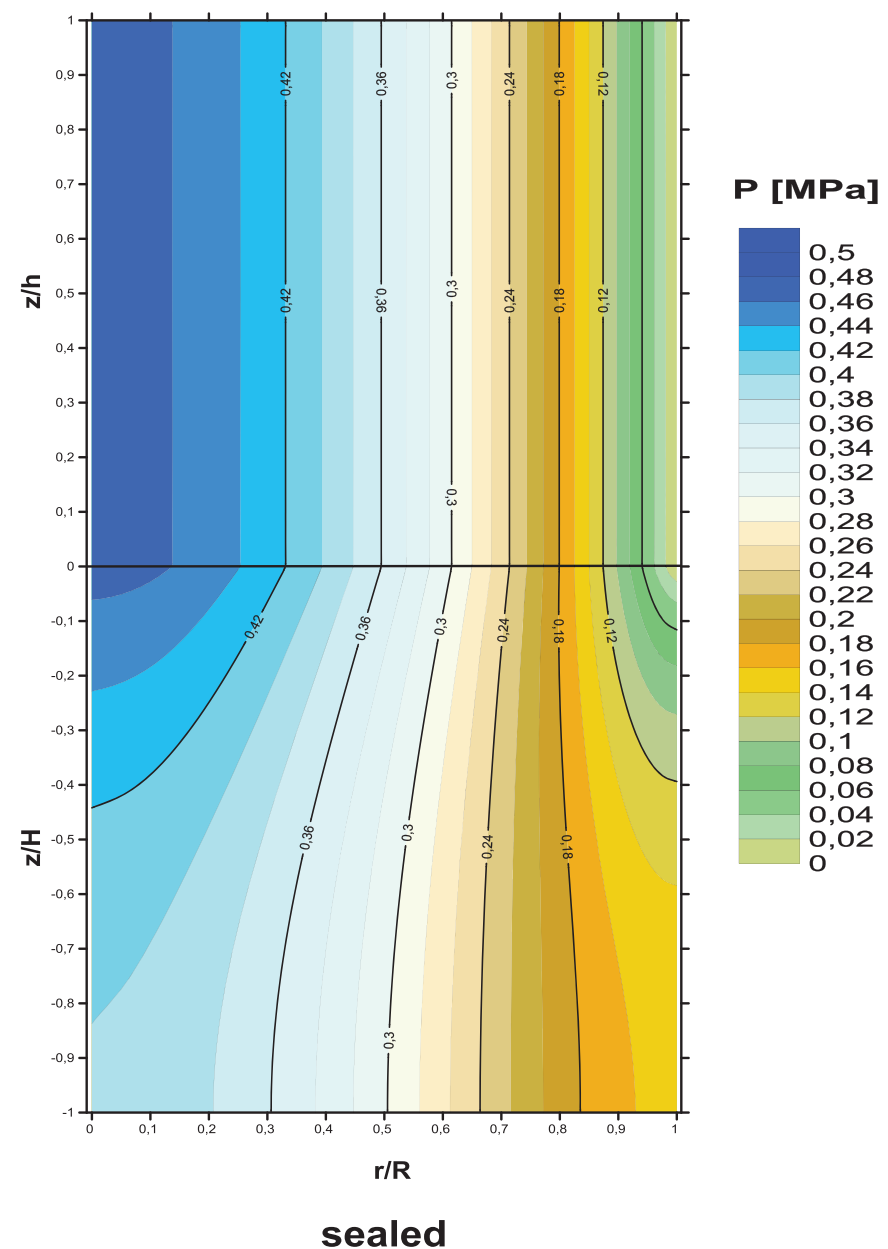

Fig. 8. Pressure contours (level lines) in film gap and anisotropic porous matrix for both sealed and unsealed configurations for $\tilde{\ell}=0.2$ and $\phi=0$ at $\tilde{\boldsymbol{t}}=0.3$.

The time variations of the central-pressure $p_{\max }$, the parietal shear stress $\tau_{r z}$ at $r=R$, the friction force $F_{f}$ and the load carrying capacity $W$ for various values of the couple stress parameter $\tilde{\ell}$ are presented in Figures $15-18$, respectively. It is shown that the all aforementioned hydrodynamic characteristics increase significantly with increasing of the couple stress parameter $\ell$ over the entire cycle. This result agrees with that of J. R. Lin [36]. Furthermore, the graphical results are always antisymmetric with respect to $\tilde{t}=0.5$ which follow the same trend as the squeeze velocity (Fig. 3). It should be noted that the peak values of these parameters are more important for the sealed case.

Figure 19 shows the influence of the couple stress parameter $\tilde{\ell}$, the leakage flow $Q$ calculated for sealed and unsealed cases during one cycle of oscillating squeeze motion.

For the sealed case, the leakage flow is not affected by the couple stress parameter since the flow that enters the porous matrix returns to the fluid layer regardless of $\tilde{\ell}$. In contrast, the leakage flow in the film gap decreases when increasing the couple stress parameter for the unsealed case. In this situation, the leak occurs both at the edge of the fluid layer and the porous matrix.
Figure 20 depicts the variations of the power loss for various values of the couple stress parameter $\tilde{\ell}$ at $\tilde{t}=0.3$. The obtained results highlight the weakness of the power loss for both sealed and unsealed configurations as well as for Newtonian and non-Newtonian couple stress fluid models.

\section{Conclusion}

This research was basically concerned with the theoretical and numerical analysis of lubrication by squeezing film effect in polluted mechanism by modelling the lubricant as a couple stress suspended fluid.

The proposed model concerns practically all systems where the squeeze film effect is the only mechanism for pressure generation, especially when a sufficiently large wedge effect is not expected [11,36-40], for example:

- the case of the internal combustion engine connectingrod small end bearing,

- the case of human hip and knee joints (for certain sporting gestures),

- the case of the squeeze film damper bearings (SFDB) used in certain rotating machinery. 

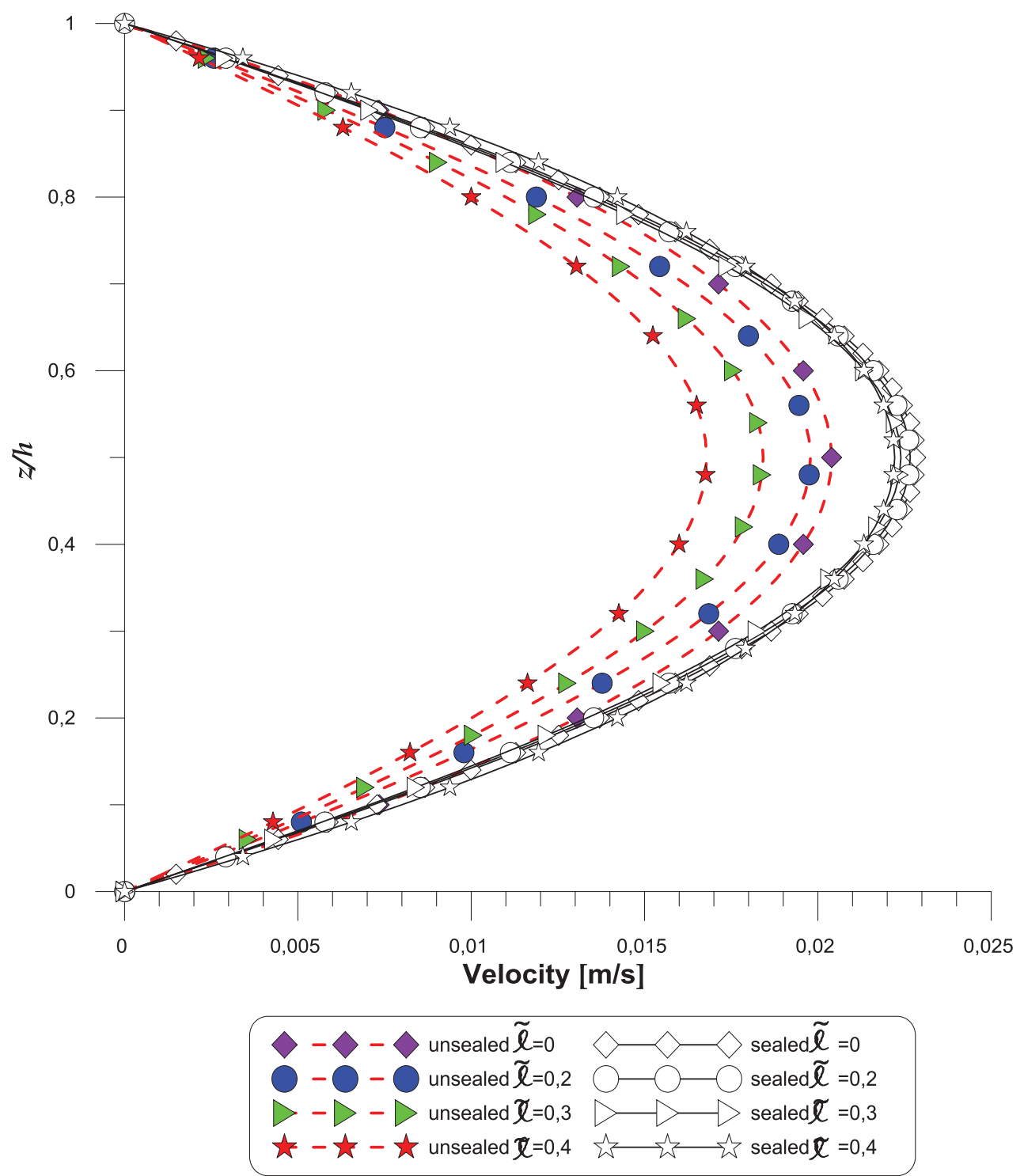

Fig. 9. Radial velocity profile at the edge $\boldsymbol{u}(\boldsymbol{r}=\boldsymbol{R})$ calculated for different values of the couple stress parameter $\tilde{\ell}$ for both sealed and unsealed configurations at $\tilde{\boldsymbol{t}}=0.3$ and for $\phi=0$.

On the basis of V. K. Stokes micro-continuum theory, the governing equations including the modified Reynolds' equation and the modified Darcy's equation were derived for laminar squeezing flow and solved numerically by the finite difference method. The nonlinear fluid-solid interaction problem for both sealed and unsealed configurations was solved numerically using a sub-relaxed iterative procedure for given values of the couple stress parameter, the permeability parameters, and the volume fraction of particles. The numerical results were graphically presented for low amplitude and frequency sinusoidal motion of the upper rigid disc taking into account directly the slip phenomenon at the porous-fluid interface. Enhancements in the squeeze film pressure and load carrying capacity (lift force) were observed for larger values of the couple stress parameter. Further for a given value of the couple stress parameter, enhancements in squeeze film pressure and load carrying capacity were obtained by increasing reasonably the volume fraction of particles in base fluid especially for the sealed porous disc configuration.

It was also found that for a given couple stress parameter the parietal shear stresses increased with increase in volume fraction of particles suspended in the base lubricant and this increase was more pronounced for the porous disc with sealed boundary. On the other hand, the side leakage flow obtained for the unsealed case decreased over the entire cycle of oscillation, i.e. for $\tilde{t}$ varying from 0 . to 1 , when increasing the couple stress parameter. However, the side leakage flow calculated in the sealed case remains unchanged for the different values of couple stress parameter.

Finally, it was shown that the power losses for both sealed and unsealed cases remain weak for all the values of couple stress parameter considered. 

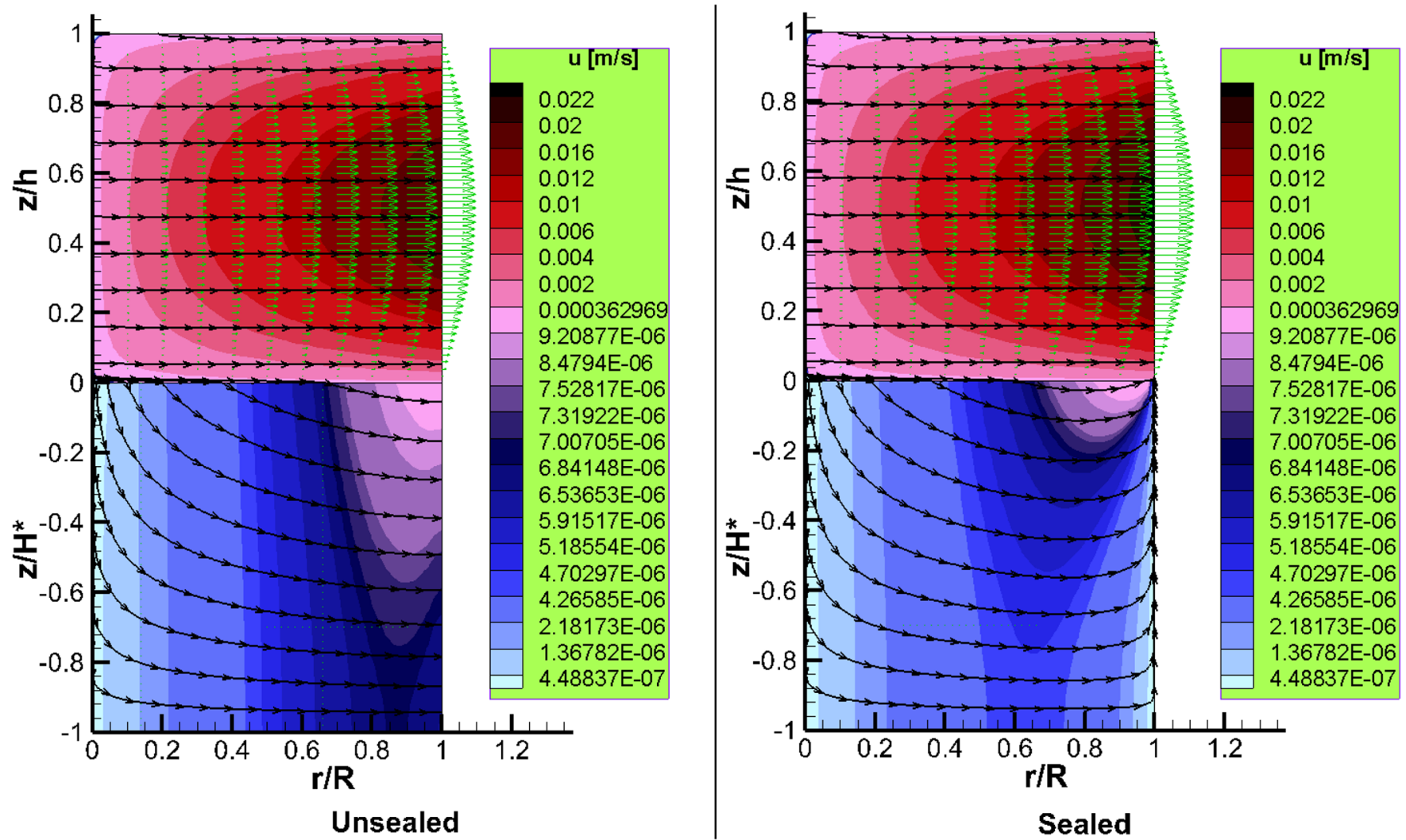

Fig. 10. Streamlines and mean velocity fields of fluid flow in film gap and anisotropic porous matrix for both sealed and unsealed configurations for $\tilde{\ell}=0.2$ and $\phi=0$ at $\tilde{\boldsymbol{t}}=0.3$.

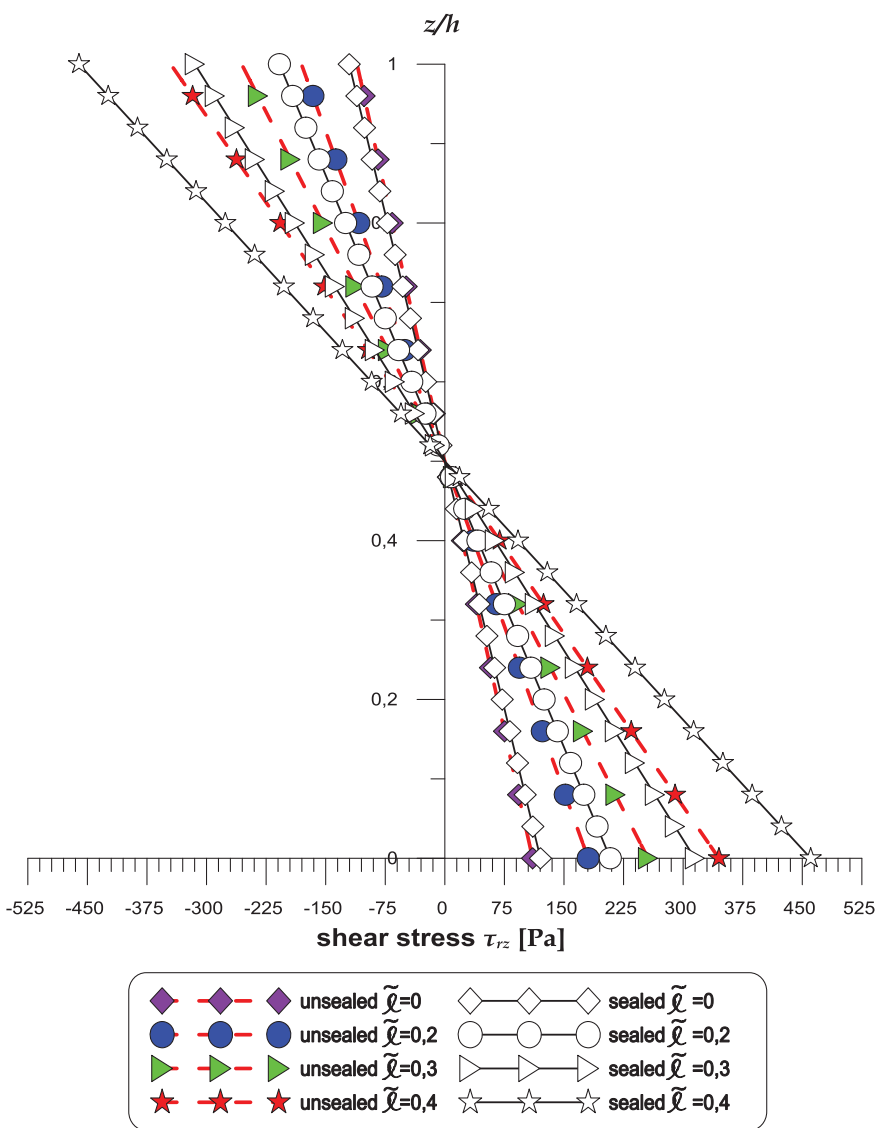

Fig. 11. Variations of shear stresses at the edge $\boldsymbol{\tau}_{\boldsymbol{r} z}(\boldsymbol{r}=\boldsymbol{R}, \boldsymbol{z})$ calculated for different values of couple stress parameter $\tilde{\ell}$ for both sealed and unsealed configurations at $\tilde{\boldsymbol{t}}=0.3$ and for $\phi=0$. 


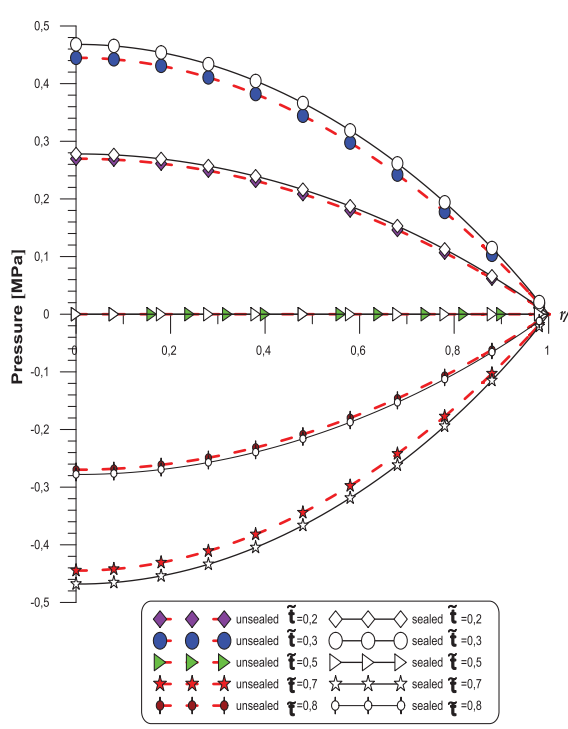

a) $\phi=0$

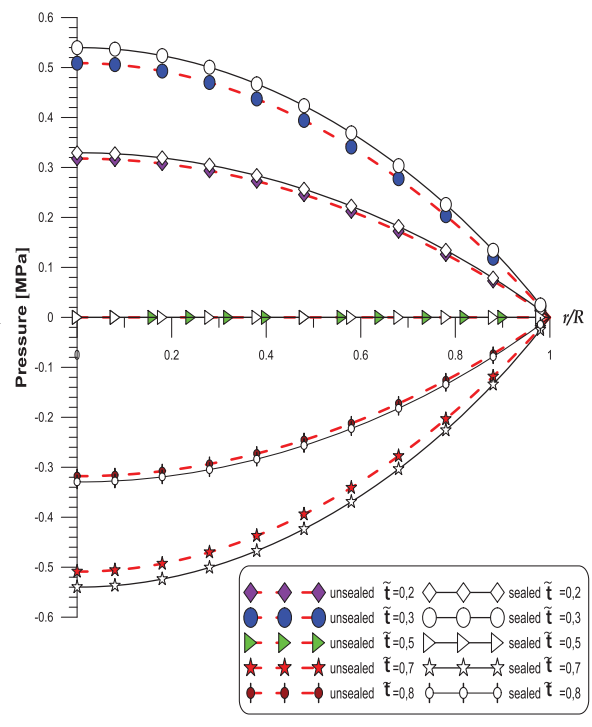

b) $\phi=0.1$

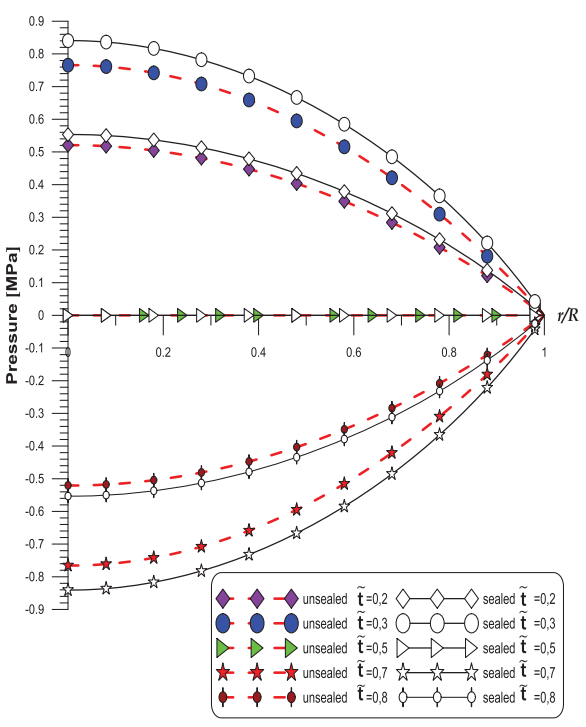

c) $\phi=0.3$

Fig. 12. Distributions of the film pressure $\boldsymbol{p}$ in one cycle of oscillations for both sealed and unsealed configurations for $\tilde{\ell}=0.2$ and different values of volume fraction.

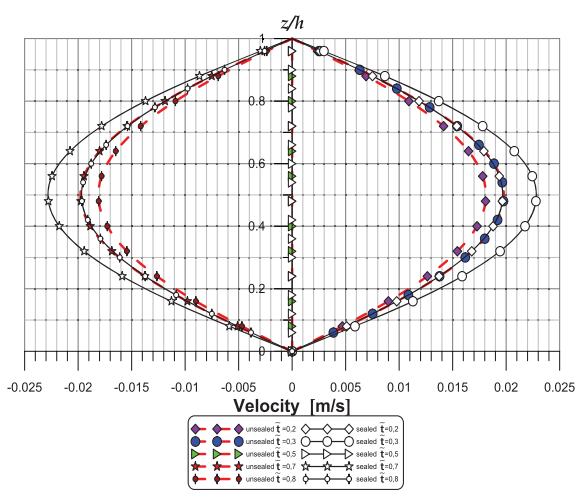

a) $\phi=0$

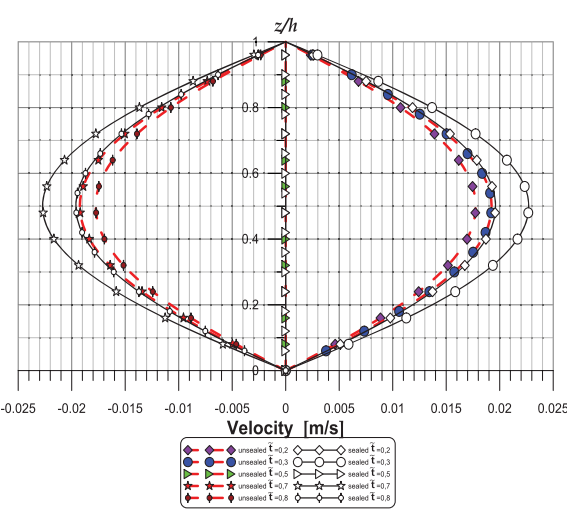

b) $\phi=0.1$

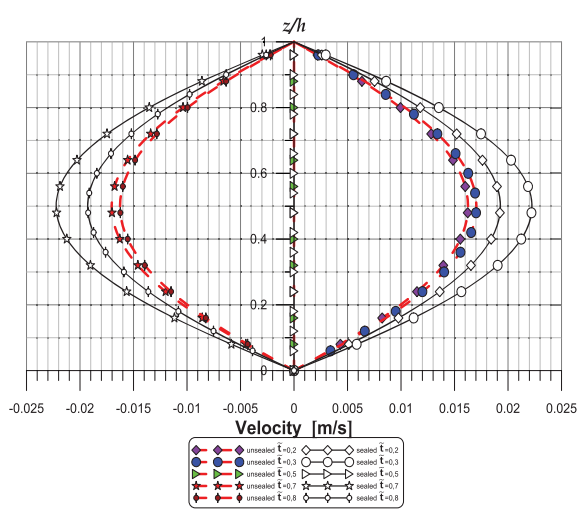

c) $\phi=0.3$

Fig. 13. Radial velocity profile $\boldsymbol{u}(\boldsymbol{r}=\boldsymbol{R})$ in one cycle of oscillation for both sealed and unsealed configurations for $\tilde{\ell}=0.2$ and different values of volume fraction.

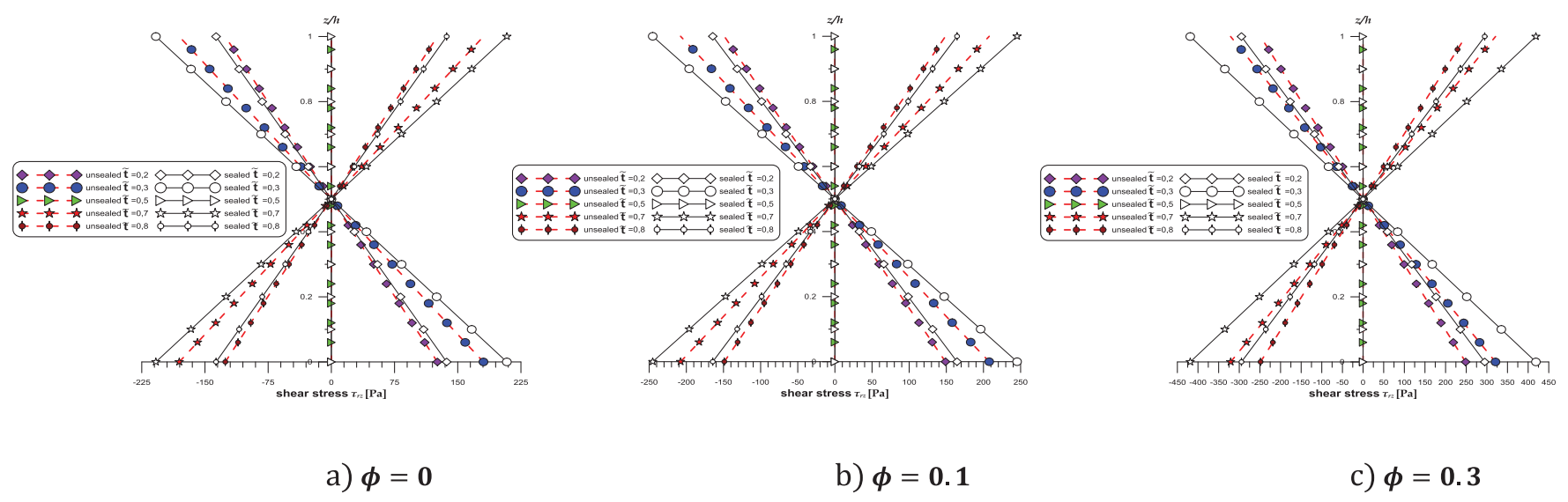

Fig. 14. Variation of shear stresses $\boldsymbol{\tau}_{\boldsymbol{r} z}(\boldsymbol{r}=\boldsymbol{R}, \boldsymbol{z})$ in one cycle of oscillation for a negative and a positive squeeze for both sealed and unsealed configurations for $\tilde{\ell}=0.2$ and different values of volume fraction. 


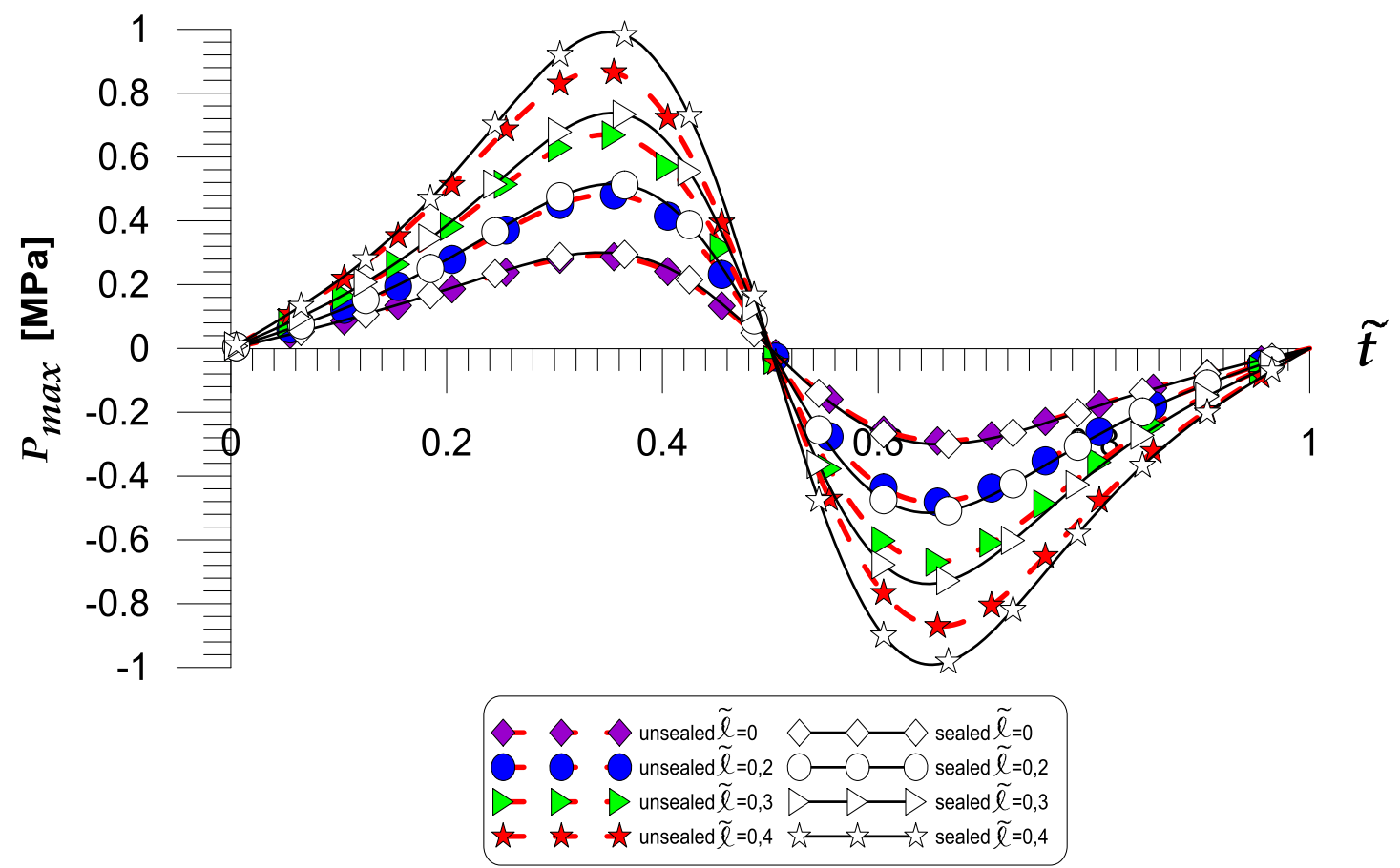

Fig. 15. Variations of the peak pressure $p_{\max }$ during one cycle of oscillation for different values of couple stress parameter $\tilde{\ell}$ in both sealed and unsealed case for $\phi=0$.

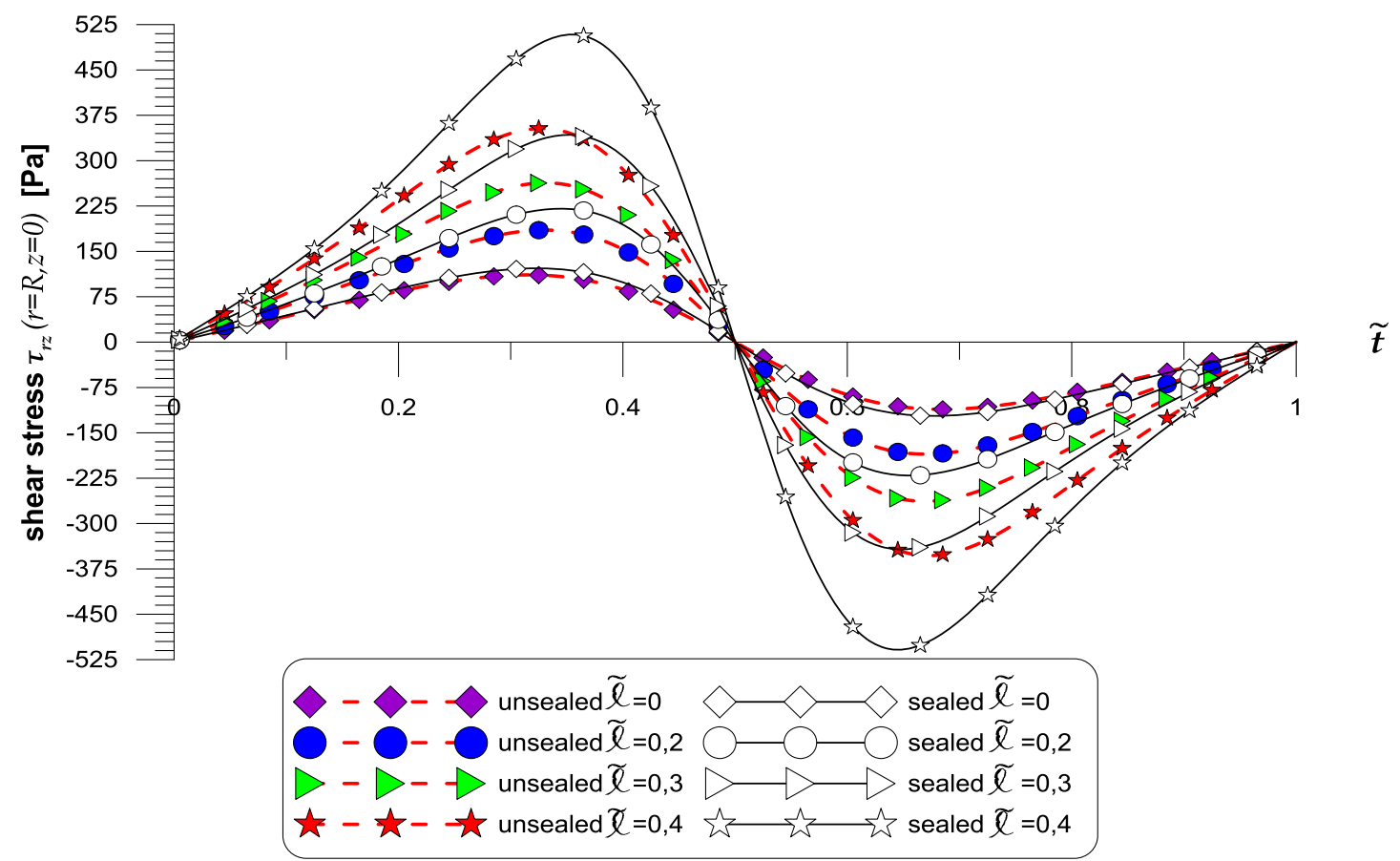

Fig. 16. Variations of the parietal shear stress at the edge $\boldsymbol{\tau}_{r \boldsymbol{z}}(\boldsymbol{r}=\boldsymbol{R}, \boldsymbol{z}=0)$ over one cycle of oscillations for different values of the couple stress parameter $\tilde{\ell}$ in both sealed and unsealed case for $\phi=0$. 


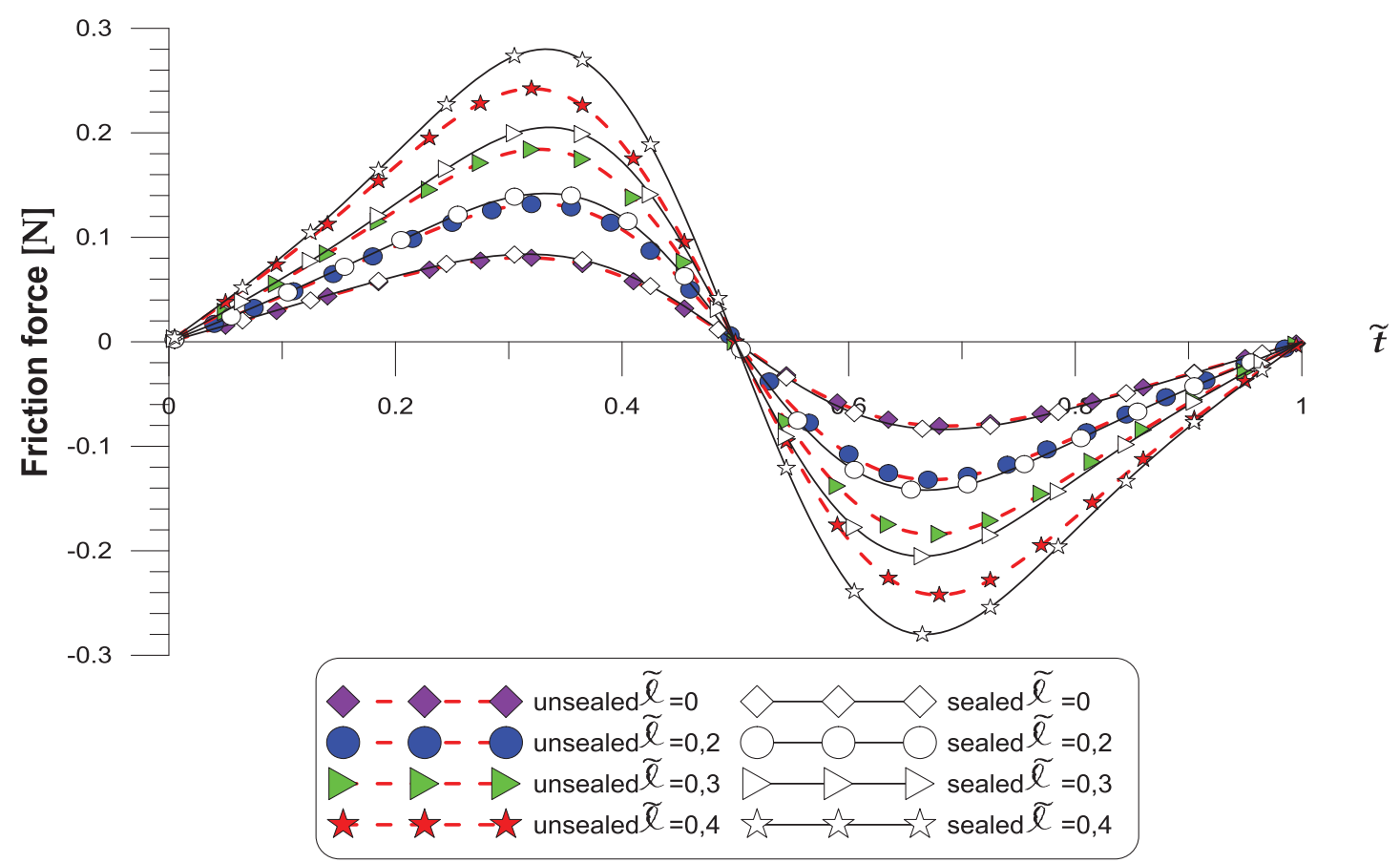

Fig. 17. Evolutions of the friction force $\boldsymbol{F}_{f}$ over one cycle of oscillations for different values of couple stress parameter $\tilde{\ell}$ in both sealed and unsealed case for $\phi=0$.

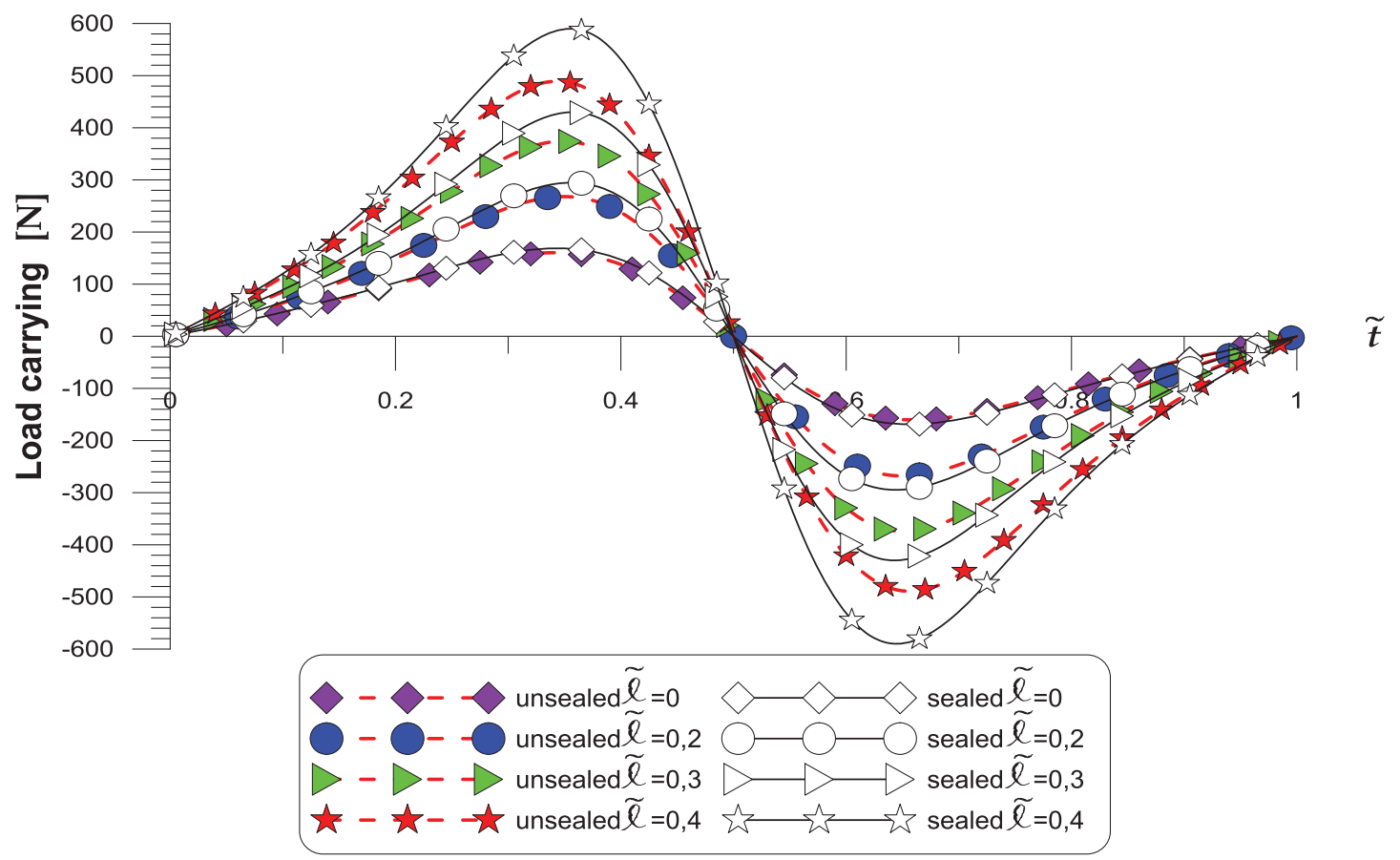

Fig. 18. Evolutions of the lift force $\boldsymbol{W}$ over one cycle of oscillations for different values of couple stress parameter $\tilde{\ell}$ in both sealed and unsealed case for $\phi=0$. 


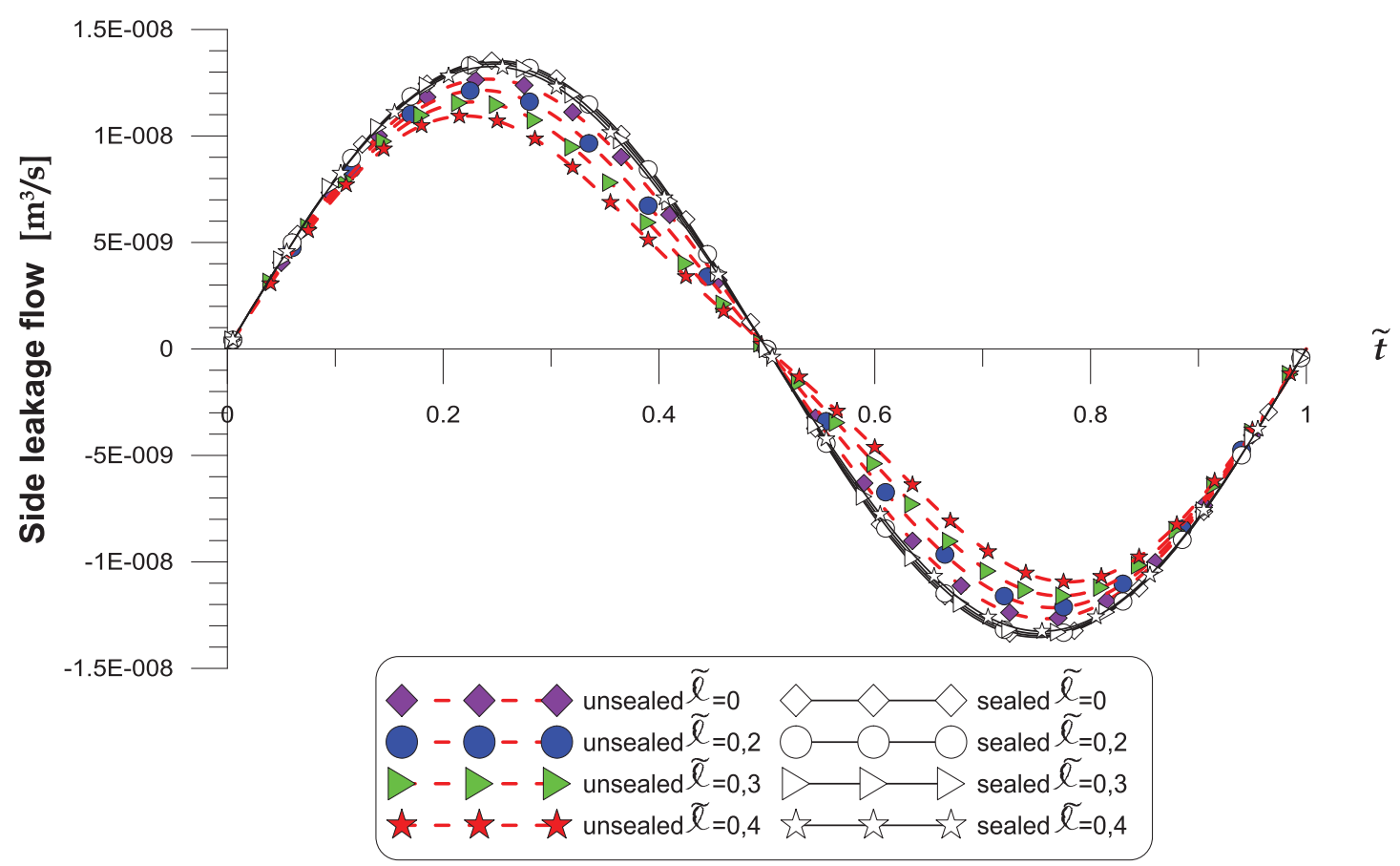

Fig. 19. Variations of the flow rate at the edge $\boldsymbol{Q}$ over one cycle of oscillations for different values of couple stress parameter $\tilde{\ell}$ in both sealed and unsealed case for $\phi=0$.

\section{Power loss W [W]}

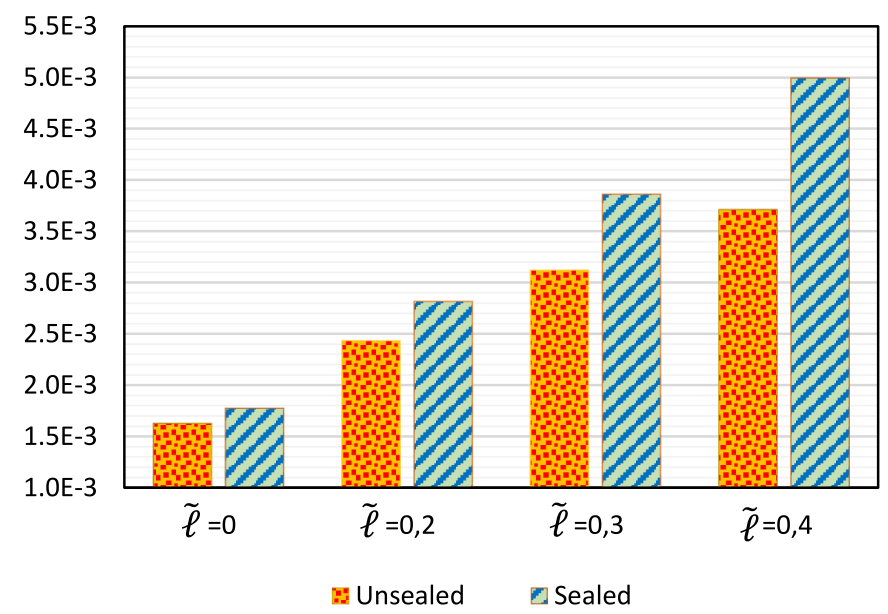

Fig. 20. Evolutions of power losses for various values of the couple stress parameter $\tilde{\ell}$ for sealed and unsealed configurations at $\tilde{\boldsymbol{t}}=0.3$ and for $\phi=0$.

\section{Nomenclature}

$F_{f} \quad$ Friction force, $[N]$

$f^{*} \quad$ Sinusoidal motion frequency, $[\mathrm{Hz}]$

$H^{*} \quad$ Porous disc thickness, [m]

$h \quad$ Film thickness, $[\mathrm{m}]$ $\tilde{h} \quad$ Non-dimensional film thickness $=\frac{h}{h_{0}},[-]$

$h_{0} \quad$ Initial film thickness, [m]

$\tilde{h}_{a} \quad$ Amplitude of sinusoidal motion, $[\mathrm{m}]$

$\tilde{h}_{a} \quad$ Non-dimensional amplitude of sinusoidal motion= $\frac{h_{a}}{h_{0}},[-]$

$\kappa_{r}, \kappa_{z}$ Permeability coefficients in the directions $r$ and $z$, respectively, $\left[\mathrm{m}^{2}\right]$

$\ell_{\tilde{\ell}} \quad$ Couple stress parameter $=\sqrt{\eta / \mu_{s}},[\mathrm{~m}]$

$\tilde{\ell} \quad$ Non-dimensional couple stress parameter $=\frac{\ell}{h_{0}},[-]$

$p \quad$ Pressure in the film region, $[\mathrm{Pa}]$

$p^{*} \quad$ Pressure in the porous region, $[\mathrm{Pa}]$

$\mathcal{P} \quad$ Power loss, $[W]$

$Q \quad$ Side leakage flow, $\left[\mathrm{m}^{3} / \mathrm{s}\right]$

$r, z \quad$ Radial and axial coordinates, $[\mathrm{m}]$

$\tilde{r} \quad$ Non-dimensional radial coordinate $=\frac{r}{R},[-]$

$R \quad$ Radius of the disc, $[\mathrm{m}]$

$t \quad$ Time, $[\mathrm{s}]$

$\tilde{t} \quad$ Non-dimensional time $=f t,[-]$

$u, w \quad$ Components of fluid velocity in $r$ and $z$ directions, respectively, $[\mathrm{m} / \mathrm{s}]$

$\langle u\rangle \quad$ Mean fluid radial velocity, $[\mathrm{m} / \mathrm{s}]$

$U_{s} \quad$ Slip velocity at the fluid-porous interface, $[\mathrm{m} / \mathrm{s}]$

$u^{*}, w^{*} \quad$ Darcy's velocity components in $r$ and $z$ directions, respectively, $[\mathrm{m} / \mathrm{s}]$

$W \quad$ Load capacity, $[N]$

$W_{s q} \quad$ Squeezing axial velocity, $[\mathrm{m} / \mathrm{s}]$

$\tilde{W}_{s q}^{s q} \quad$ Non-dimensional squeezing velocity $=\frac{W_{s q}}{f h_{0}},[-]$

$\mu_{s} \quad$ Effective viscosity of suspension, [Pa.s]

$\mu_{b f} \quad$ Viscosity of base fluid, [Pa.s]

$\phi \quad$ Volume fraction of particle, $[-]$ 
$\alpha_{r}, \alpha_{z}$ Permeability parameters of the anisotropic porous matrix in the $r$ and $z$ directions, respectively, $\left[\mathrm{m}^{4} /\right.$ N.s $]$

$\beta_{r}, \beta_{z} \quad$ Ratios of microstructure size to pore size, [-]

$\eta \quad$ Material constant responsible for the couple stress property, [N.s]

$[\bar{\eta}] \quad$ Intrinsic viscosity,[Pa.s]

$\tau_{r z} \quad$ Shear stress, $[\mathrm{Pa}]$

The authors are thankful to the anonymous reviewer for his valuable comments on the earlier draft of this paper.

\section{Appendix A: Morgan-Cameron approximation [13]}

Integrating the Darcy's equation (10), written for an isotropic porous medium (i.e. $\alpha_{r}=\alpha_{z}$ )with respect to $z$-variable over the porous layer thickness, and using the boundary conditions of solid backing (13d), we obtain

$$
\left.\frac{\partial p^{*}}{\partial z}\right|_{z=0}=-\int_{-H^{*}}^{0} \frac{1}{r} \frac{\partial}{\partial r}\left(r \frac{\partial p^{*}}{\partial r}\right) d z .
$$

Assuming that the porous layer thickness, $H^{*}$ is very small compared to disc radius and using the pressure continuity condition (13c) at the fluid film-porous layer interface $(z=0)$, equation (A.1) becomes

$$
\left.\frac{\partial p^{*}}{\partial z}\right|_{z=0}=-H^{*} \frac{1}{r} \frac{\partial}{\partial r}\left(r \frac{\partial p}{\partial r}\right)
$$

Then, the modified Darcy's velocity component at the interface $(z=0)$ is given by

$$
\left.w^{*}\right|_{z=0}=-\left.\alpha_{z} \frac{\partial p^{*}}{\partial z}\right|_{z=0}=\alpha_{z} H^{*} \frac{1}{r} \frac{\partial}{\partial r}\left(r \frac{\partial p}{\partial r}\right) .
$$

Taking into account the relationship (A.3), the modified Reynolds' equation (17) when the slip velocity at the interface $(z=0)$ is ignored reduces to

$$
\frac{1}{r} \frac{\partial}{\partial r}\left(r \frac{\partial p}{\partial r}\right)=\frac{12 \mu_{s} \frac{d h}{\mathrm{~d} t}}{G(h, l)+12 \mu_{s} \alpha_{z} H^{*}} .
$$

Using the boundary conditions on the fluid-film pressure $p(13 \mathrm{a})$ and $(13 \mathrm{~b})$, the analytical solution of equation (A.4) is derived as

$$
p(r, t)=\frac{3 \mu_{s} \frac{d h}{\mathrm{~d} t}\left(r^{2}-R^{2}\right)}{G(h, l)+12 \mu_{s} \alpha_{z} H^{*}} .
$$

\section{References}

[1] O. Reynolds, On the theory of lubrication and its application to Mr. Beauchamp tower's experiments, including an experimental determination of the viscosity of olive oil, Phil. Trans. R. Soc. London 177, 157-234 (1886)

[2] J.D. Jackson, A study of squeezing flow, Appl. Sci. Res. Sect. A 11, 148-152 (1963)

[3] N. Bujurke, M. Jagadeeswar, P. Hiremath, Analysis of normal stress effects in a squeeze film porous bearing, Wear 116, 237-248 (1987)

[4] N. Bujurke, N. Naduvinamani, A note on squeeze film between rough anisotropic porous rectangular plates, Wear 217, 225-230 (1998)

[5] N. Bujurke, R. Kudenatti, Surface roughness effects on squeeze film poroelastic bearings, Appl. Math. Comput. 2, 1181-1195 (2006)

[6] M. Megat Ahmad, D. Gethin, T. Claypole, B. Roylance, Numerical and experimental investigation into porous squeeze films, Tribol. Int. 31, 189-199 (1998)

[7] M. Hlaváček, Squeeze-film lubrication of the human ankle joint with synovial fluid filtrated by articular cartilage with the superficial zone worn out, J. Biomech. 33, 1415-1422 (2000)

[8] A. Ruggiero, E. Gòmez, R. D'Amato, Approximate analytical model for the squeeze-film lubrication of the human ankle joint with synovial fluid filtrated by articular cartilage, Tribol. Lett. 41, 337-343 (2011)

[9] M. Alshehri, S.K. Sharma, Computational Model for the Generalised Dispersion of Synovial Fluid, Int. J. Adv. Comput. Sci. Appl. 8, 2 (2017)

[10] B.J. Hamrock, Fundamentals of Fluid Film Lubrication, National Aeronautics and Space Administration, Madison, OH, 1990

[11] Y. Hori, Hydrodynamic Lubrication, Springer, Tokyo, 2006

[12] P. Jurczak, Pressure distribution in a squeeze film biobearing lubricated by a synovial fluid, Int. J. Appl. Mech. Eng. 11, 857-864 (2006)

[13] V. Morgan, A. Cameron, Mechanismus of lubrication in porous metal bearing, Proceedings, Conference on Lubrication and Wear, The Institution of Mechanical Engineers, London, pp. 151-157, 1957

[14] J.-R. Lin, L.-M. Chu, W.-L. Li, R.-F. Lu, Combined effects of piezo-viscous dependency and non-Newtonian couple stresses in wide parallel-plate squeeze-film characteristics, Tribol. Int. 44, 1598-1602 (2011)

[15] M. Nabhani, M. El Khlifi, B. Bou-Saïd, Non-Newtonian couple stress poroelastic squeeze film, Tribol. Int. 64, 116127 (2013)

[16] V. Ponnuswamy, S. Govindaraj, Behaviour of couple stress fluids in porous annular squeeze films, J. Appl. Math. Phys. 2, 349-358 (2014)

[17] G.S. Beavers, D.D. Joseph, Boundary conditions at a naturally permeable wall, J. Fluid Mech. 30, 197-207 (1967)

[18] H.C. Brinkman, A calculation of the viscous force exerted by a flowing fluid on a dense swarm of particles, Flow Turbul. Combust. 1, 27 (1949)

[19] D.A. Nield, A. Bejan, Convection in Porous Media, 5th edn., Springer, Cham, 2017 
[20] A. Ruggiero, E. Gómez, R. D'Amato, Approximate closedform solution of the synovial fluid film force in the human ankle joint with non-Newtonian lubricant, Tribol. Int. 57, $156-161$ (2013)

[21] V.K. Stokes, Couple stresses in fluids, Phys. Fluids (1966)

[22] M. Devekar, T.K.V. Iyengar, Stokes' problems for an incompressible couple stress fluid., Nonlinear Anal. Model. Control 1, 181-190 (2008)

[23] V.K. Stokes, Theories of Fluids with Microstructure: An introduction, Springer-Verlag, Berlin, 1984

[24] I.M. Krieger, T.J. Dougherty, A Mechanism for NonNewtonian Flow in Suspensions of Rigid Spheres, J. Rheol. 3, 137 (1959)

[25] C. Haisheng, D. Yulong, T. Chunqing, Rheological behaviour of nanofluids, New J. Phys. 9, 367 (2007)

[26] I. Mahbubul, R. Saidur, M. Amalina, Latest developments on the viscosity of nanofluids, Int. J. Heat Mass Transf. 55, 874-885 (2012)

[27] M. Kole, T. Dey, Effect of aggregation on the viscosity of copper oxide-gear oil nanofluids, Int. J. Therm. Sci. 50, 1741 (2011)

[28] R. Roscoe, The viscosity of suspensions of rigid spheres, Br. J. Appl. Phys. 3, 8 (1952)

[29] E.R. Booser, CRC Handbook of Lubrication and Tribology, CRC Press, Boca Raton, Florida, USA, 1994, 3

[30] B.J. Hamrock, S.R. Schmid, B.O. Jacobson, Fundamental of fluid film lubrication, 2nd edn., Boca Raton, Florida, USA, 2004

[31] N.B. Naduvinamani, A. Siddangouda, Combined effects of surface roughness and couple stresses on squeeze film lubrication between porous circular stepped plates, J. Eng. Tribol. 221, 525-534 (2007)

[32] N.B. Naduvinamani, S.T. Fathima, P.S. Hiremath, Effect of surface roughness on characteristics of couplestress squeeze film between anisotropic porous rectangular plates, Fluid Dyn. Res. 32, 5 (2003)

[33] A.E. Yousif, A.A. Al-allaq, The hydrodynamic squeeze film lubrication of the ankle joint, Int. J. Mech. Eng. Appl. 1, 34$42(2013)$

[34] J.D. Hoffman, S. Frankel, Numerical Methods for Engineers and Scientists, 2nd edn., CRC Press, Boca Raton, Florida, USA, 2001
[35] M. Nabhani, M. El Khlifi, B. Bou-Saïd, Combiend nonNewtonian and viscous shear effects on porous squeeze film behaviour, Tribol. Trans. 55, 491-502 (2012)

[36] J.R. Lin, "Oscillating circular squeeze films considering the combined effects of fluid inertial forces and non-Newtonian couple stresses.," Proc. IMecE, Journal of Enginnering Tribology, Part J., vol. 222, 2008.

[37] J.R. Lin, Oscillating circular squeeze films considering the combined effects of fluid inertial forces and non-Newtonian couple stresses, Proc. Inst. Mech. Eng. J. Eng. Tribol. J. 222, 7 (2008)

[38] N.B. Naduvinamani, P.S. Hiremath, G. Gurubasavaraj, Squeeze film lubrication of a short porous journal bearing with couple stress fluids., Tribol. Int. 34, 739-747 (2001)

[39] O.S.T. Gbehe, M. El Khlifi, M. Nabhani, B. Bou-Saïd, Investigation of couple stress effects on poroelastic squeeze film of parallel plates, Lubrication Sci. 29, 93-113 (2016)

[40] F.A. Rodrigues, Etude de la dynamique non lineaire d'amortisseurs du type squeeze film, Ecole Centrale de Lyon, Lyon, 2003

[41] J.H. Dumbleton, Tribology of natural and artificial joints, Elsevier, New York, 1981

[42] D.J. Knox, S.K. Wilson, B.R. Duffy, S. McKee, Squeeze-Film Flow in the Presence of a Thin Porous Bed, with Application to the Human Knee Joint, Oxford Centre for Collaborative Applied Mathematics, 2012

[43] M. Yousfi, B. Bou-Saïd, J. Tichy, Axisymmetric squeezing of a Phan-Thien and Tanner lubricant film under imposed constant load in the presence of a poroelastic medium, Lubrication Sci. 27, 505-522 (2015)

[44] D.D. Frisbie, M.W. Cross, C.W. McIlwraith, A comparative study of articular cartilage thickness in the stifle of animal species used in human pre-clinical studies compared to articular cartilage thickness in the human knee, Vet. Comp. Orthop. Traumatol. 19, 142-146 (2006)

[45] V.C. Mow, A. Ratcliffe, A. Poole, Cartilage and diarthrodial joints as paradigms for hierarchical materials and structures, Biomaterials 13, 67-97 (1992)

[46] J. Katta, Z. Jin, E. Ingham, J. Fisher, Biotribology of articular cartilage-A review of the recent advances, Med. Eng. Phys. 30, 1349-1363 (2008)

Cite this article as: B. Boussaha, M. Lahmar, B. Bou-Said, H. Boucherit, Non-Newtonian couple-stress squeeze film behaviour between oscillating anisotropic porous circular discs with sealed boundary, Mechanics \& Industry 21, 311 (2020) 